NATIONAL RESEARCH UNIVERSITY HIGHER SCHOOL OF ECONOMICS

Olga Rubtsova, Elena Gorbunova

\title{
THE EFFECT OF CATEGORICAL SUPERIORITY IN SUBSEQUENT SEARCH MISSES
}

\author{
BASIC RESEARCH PROGRAM \\ WORKING PAPERS
}

SERIES: PSYCHOLOGY

WP BRP 119/PSY/2020

This Working Paper is an output of a research project implemented within NRU HSE's Annual Thematic Plan for Basic and Applied Research. Any opinions or claims contained in this Working Paper do not necessarily reflect the 


\title{
THE EFFECT OF CATEGORICAL SUPERIORITY IN SUBSEQUENT SEARCH MISSES ${ }^{2}$
}

\begin{abstract}
Subsequent search misses refer to the decrease in accuracy of second target detection in dualtarget visual search. One of the theoretical explanations of SSM errors is similarity bias - the tendency to search for similar targets and to miss the dissimilar ones. The current study focuses on both perceptual and categorical similarity and their individual roles in SSM. Three experiments investigated the role of perceptual and categorical similarity in subsequent search misses, wherein perceptual and categorical similarity were manipulated separately, and task relevance was controlled. The role of both perceptual and categorical similarity was revealed, however, the categorical similarity had greater impact on second target detection.
\end{abstract}

Keywords: visual attention, visual search, subsequent search misses, perceptual similarity, categorical similarity

JEL Classification: Z

\footnotetext{
${ }^{1}$ National Research University Higher School of Economics, Laboratory for Cognitive Psychology of Digital Interfaces Users

${ }^{2}$ National Research University Higher School of Economics, School of Psychology

2 This Working Paper is an output of a research project implemented within NRU HSE's Annual Thematic Plan for Basic and Applied Research. Any opinions or claims contained in this Working Paper do not necessarily reflect the views of HSE.
} 


\section{Introduction}

Visual search, which is one of the most common paradigms in visual science, implies searching for specific objects, called "targets", among irrelevant objects, called "distracters". There are many various studies concerning searching both for one and for several targets among distracters, and the latter scenario is particularly interesting, since it resembles our daily visual search activities. Mechanisms for searching for multiple targets are still not very clear, since more and more effects are being discovered in this area of visual search. One of such effects is called "subsequent search misses", or SSM, and it indicates the reduction in accuracy of finding the second target after finding the first target (e.g. Adamo, Cain, Mitroff, 2013). SSM is the term that appeared not so long ago, replacing the term "satisfaction of search", which initially came from practical fields, such as radiology. This effect was not very precisely studied back then, but with the development of research on visual search it finally came around late 2000's. A series of experiments held by M. Fleck and his colleagues (Fleck et al., 2010) showed the emergence of SSM in laboratory conditions. In these experiments Fleck and colleagues used specific stimuli that later on became the main experimental material for studying SSM. They used letters ' $\mathrm{T}$ ' as targets and letters ' $L$ ' as distracters. The letters had various orientations and salience. They managed to prove that the accuracy of search for a target declines in case when it the second target presented on set, in comparison with searching for only one target. Since then quite a few additional studies have been conducted in order to further investigate the mechanisms responsible for these mistakes in visual search. In most recent studies this effect is discussed with regard to typical real-world situations, such as driving (Sall, Feng, 2019) and airport security screening (Biggs et al, 2018). Therefore, studying this effect is of particular importance, and the experimentally verified data may be ecologically valid.

Several theories are provided to explain the effect of SSM. One of these theories derived from radiology and claimed that satisfaction of search takes place when multiple targets are present. When radiologists successfully identified one target, they were satisfied with it and were not enough motivated to search further (Tuddenham, W. J. 1962). However, this hypothesis met quite a few arguments against the existence of "satisfaction", mainly because searchers usually continue looking for additional targets, even after successfully finding one (Berbaum et al., 1991, Fleck et al., 2010). With the exploration of the mechanisms responsible for this additional search appeared new theories, involving more complex explanations for SSM. One of the most popular ones is the theory of exhaustion of cognitive resources, especially those of working memory and attention. Most of the resources are involved in the processing of the first target, which leaves much less resources for the search of the second one. For example, Cain \& Mitroff (2013) study demonstrated that the accuracy of finding the second target increases, when the firstly detected 
target disappears from the screen. That might be due to the less resources left, since the spatial position of the first target needs to be kept in memory.

One of the leading explanations of SSM involves similarity bias or perceptual set. Searchers are very likely to become biased towards targets that share similar perceptual features with the initially found target. It has been argued that implicit short-term memory guides attention towards those objects that had some value recently (Kristjannson, 2010). However, there is still an active discussion, regarding the initial causes of such guidance. The evidence for the assumption of perceptual set may be found in many recent studies on SSM. For instance, in a study by Gorbunova (2017) the degree of perceptual similarity of targets was determined by targets shape, color, size and orientation. It was shown that the more features two targets shared, the more accurate the participants were identifying those targets. That provides a strong argument in favor of perceptual set theory. However, the exact mechanism of perceptual set is not completely clear. One possibility may be bottom-up perceptual priming. Another is top-down working memory guidance. Assuming the latter mechanism, is it possible to propose the hybrid SSM errors explanation, based both on similarity bias and resource depletion. The first-found target representation may be stored in working memory, causing the resource depletion and similarity bias, based on working memory guidance, at the same time.

Conceptual, or categorical, set seems to explain the mechanisms of visual search for several targets as well as perceptual set. The concept of a specific object, that is stored in our memory, can guide our attention in a visual search task. That was demonstrated, for example, in a study, where the participants searched for a teddy bear, without necessarily knowing the exact appearance of a looked-for item (Yang, Zelinsky, 2009). These results suggest that searchers could anchor their searching strategies to particular features of an abstract object (a teddy bear is something that is fluffy, has round ears, short tail, etc.), so that so-called priming takes place. Moreover, the search for categorically specified items could be explained by the spreading activation theory, which implies that attention would reach the concepts, similar to recently activated one, easier and faster (Collins, Loftus, 1975). One particular experiment involved the subjects searching for odd or even digits, and it was revealed that the accuracy of search for two targets was significantly higher in case of identical digits (Lanina \& Gorbunova, 2018). The results present evidence for collective impact of perceptual and categorical similarity of targets in SSM, although it is quite difficult to isolate individual influence of each of these factors in this particular experiment.

Interesting results were obtained by A. Biggs and colleagues in their big data research (Biggs et al, 2015). They used the mobile application Airport scanner in order to analyze the specifics of visual search for multiple targets with similarities. One of the main advantages of this work is that the ecologically valid data was used. The authors analyzed how people searched 
for real world objects, particularly forbidden items in scanned luggage. The results supported impact of both conceptual and perceptual biases in visual search. What is even more interesting, is the fact that the results also revealed the superiority of conceptual factors in comparison to perceptual factors for the reduction of SSM. However, the possible limitation of this study include that it was the big data study, but not the experiment with the controlled variables. Another point might be the real world objects used as the stimuli. Of course, this is a huge point for the ecological validity, however, it comes with the possible confounds.

The purpose of the current research was to experimentally verify the role of both categorical and perceptual similarity of targets in the emergence of subsequent search misses. Based on the previous studies, we expected to observe the effect of categorical target similarity superiority. In order to do that, we tried to experimentally separate the mentioned types of target similarity, which brings novelty in comparison to the prior research on the effect of SSM. The separate investigation of categorical and perceptual similarity would not only expand the range of factors known to influence on SSM errors. The important point would be to get closer to understanding the mechanisms of similarity bias - is it bottom-up priming or top-down guidance. If the categorical target similarity superiority (independent of perceptual similarity) would be observed, top-down guidance is more likely to be the mechanism of similarity bias.

\section{Experiment 1}

\section{Method}

\section{Participants}

30 volunteers participated in this experiment for the class credit, all students from National Research University Higher School of Economics, with normal or corrected to normal vision, without any neurological or psychological problems and native Russian speakers. Results from 2 participants were excluded due to misunderstanding the instruction, so data from 28 subjects were analyzed. Participants were 4 males and 24 females, and their age varied from 17 to $24(\mathrm{M}=18.71, \mathrm{SD}=1.74)$.

\section{Stimuli}

The letters of the Russian alphabet were used as stimuli in this experiment. Overall, 20 letters were used, and the rest were excluded due to the size and shape differences in comparison with the chosen ones. All the stimuli had the size of $3.68^{\circ} * 4.93^{\circ}$. The letters could be light gray (CIE: .313, .329, 14.2), mid-gray (CIE: .313, .329, 10.1) or dark-gray (CIE: .313, .329, 5.8), and they were always presented on a plain grey screen (CIE: .313, .329, 22.0). The way of writing of the letters was varied: it could be either uppercase or lowercase. Vowels were chosen as targets, and consonants were chosen as distracters. The perceptual similarity of the stimuli 
was determined by the way of writing - uppercase or lowercase ( $\mathrm{A}$ and $\mathrm{E}$ or $\mathrm{A}$ and $\mathrm{e}$ ), the categorical similarity was determined by the letter identity (A and $\mathrm{E}$ or $\mathrm{E}$ and $\mathrm{E}$ ). 7 experimental conditions were created in order to vary the degree of target similarity: one target with high salience, one target with low salience, no targets, two identical targets, two targets with categorical similarity, two targets with perceptual similarity, two targets with no similarity. Each trial always consisted of 20 stimuli randomly distributed on the screen, positions were set with $10 \times 5$ invisible grid. There could be 2,1 or none targets in one trial. In dual-target trials, one target was high salient and one was low salient. At the bottom of the screen two buttons (Ok and No) were presented in each trial. They both had the size of $5.83^{\circ} \times 4.93^{\circ}$ and were used by the participants to report the presence or absence of targets in certain conditions. The example of a trial is presented in Fig.1.

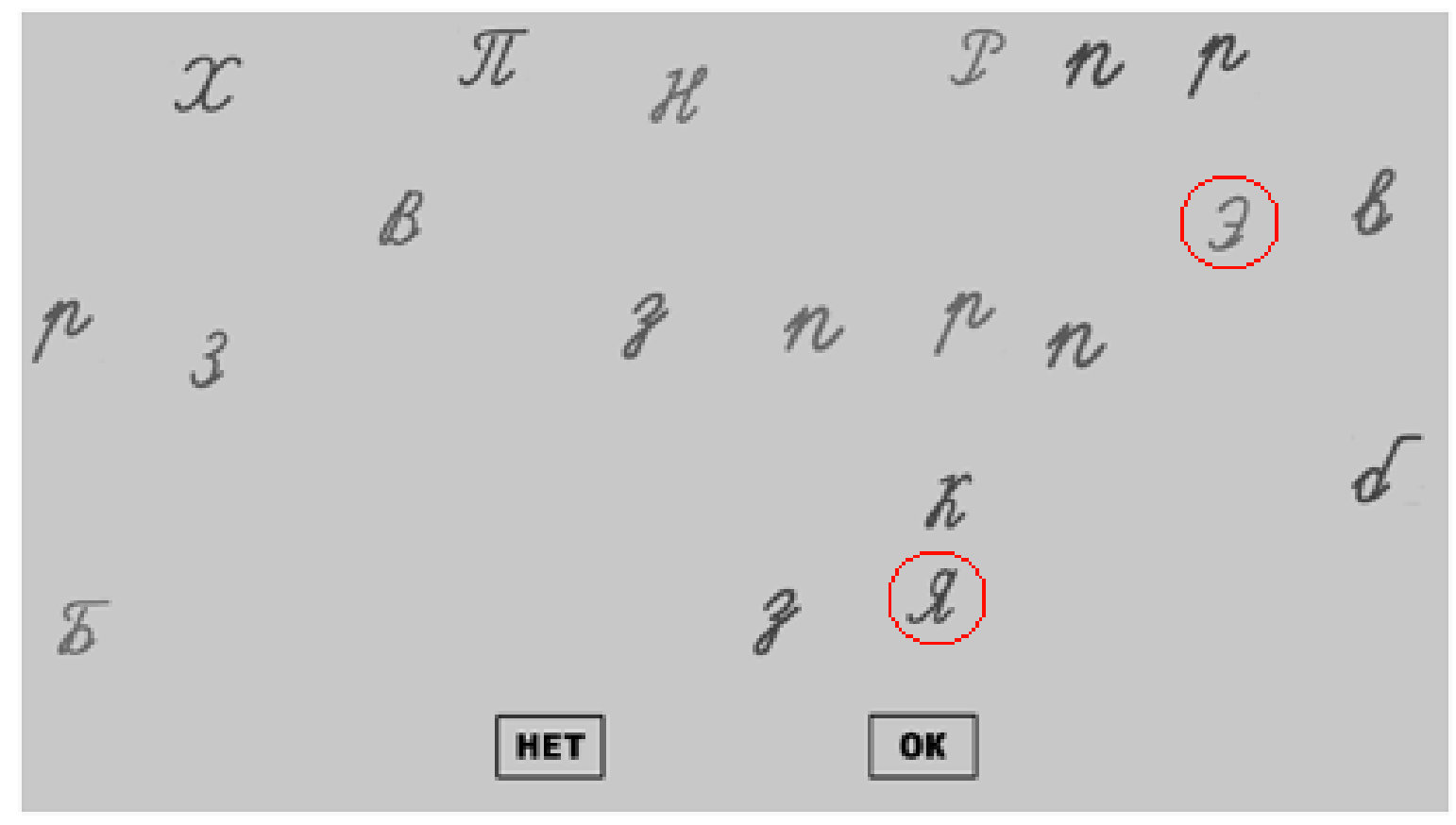

Figure 1. An example of experimental trial in Experiment 1 (condition for two targets, with perceptual but not categorical similarity - targets are marked with red circles)

The participants sat in front of BENQ-GL2250 monitor (screen resolution 1920x1080; refresh rate $76 \mathrm{~Hz}$ ) with $40 \mathrm{~cm}$ distance between their eyes and the screen. PsychoPy v. 1.90 .2 was used to present the stimuli. The participants used a standard computer mouse and a standard keyboard in order to report their answers.

\section{Procedure}

The participants were instructed to find all vowels presented on the screen. They were informed that there could be 2,1 or none targets in one trial. They used the computer mouse in order to identify targets. They had to click on the vowel as soon as they found it on the screen. In case of two targets, they clicked on the vowels consequently. In case the participants realized that there was only one vowel, they had to make the second click on the button "Ok". If they did 
not find any vowels at all, they had to click twice on the button "No". Thus, participants always made two clicks with the mouse in each trial. In order to start a new trial, the participants needed to press space bar on the keyboard. They could take some time and rest before starting a new trial each time. Altogether, the experiment consisted of 350 trials, 50 trials for each of the experimental conditions. The presentation order of trials was randomly interleaved for each participant.

Before the main part of the experiment there was a training session, which consisted of 10 trials and included different experimental conditions. Therefore, the participants could adjust to the stimuli and ask questions if they had any difficulties or misunderstanding.

\section{Results}

Accuracy and reaction time for conditions with two targets (with four different conditions of target similarity) and one low salient target were analyzed. Conditions with one target that had high salience and with no targets were irrelevant to the experimental hypotheses and were used to control the participants' attention towards the instruction, and data from those conditions were not analyzed. The analysis was made using SPSS 20.0.

Two types of analysis were made. One-way repeated measures ANOVA (factor - type of target, five levels: one target, two dissimilar targets, two perceptually similar targets, two categorically similar targets, two perceptually and categorically similar targets) was applied to reveal the presence of SSM effect if different conditions. Two-way repeated measures ANOVA for dual-target conditions, factors - perceptual similarity (present or absent) and categorical similarity (present or absent) was applied to reveal the impact of categorical and perceptual similarity for SSM errors. Pairwise comparisons were used with Holm-Bonferroni adjustment. The Greenhouse-Geisser corrections were applied, when Mauchly's sphericity tests were significant.

For accuracy, traditional SSM error analysis was performed: in conditions with two targets the percentage of correct responses was calculated only for those trials, where the second successfully identified target had low salience. Trials with low salient target identified first were not included in further analysis. Reaction time analysis was made only for correct response trials and assumed comparing the time it took to find each target for different conditions.

\section{Accuracy}

One-way repeated measures ANOVA revealed the significant impact of target similarity factor, $F(3,74)=13.231 ; p<.001 ; \eta p^{2}=.329$. Pairwise comparisons with Holm-Bonferroni adjustment revealed significant differences between following conditions: one target and two identical targets, one target and two targets with no similarity, two identical targets and two targets with perceptual similarity, two identical targets and two targets with no similarity, two 
targets with categorical similarity and two targets with no similarity, two targets with perceptual similarity and two targets with no similarity. Results are presented in Fig.2-3 and Table 1.

Two-way repeated measures ANOVA revealed the significant effect of perceptual similarity factor, $F(1,27)=15.045 ; p=.001 ; \eta p^{2}=.358$. The effect of categorical similarity was also significant, $F(1,27)=21.865 ; p<.001 ; \eta p^{2}=.447$. The interaction was not significant, $F$ $(1,27)=2.709 ; p=.111 ; \eta p^{2}=.091$.

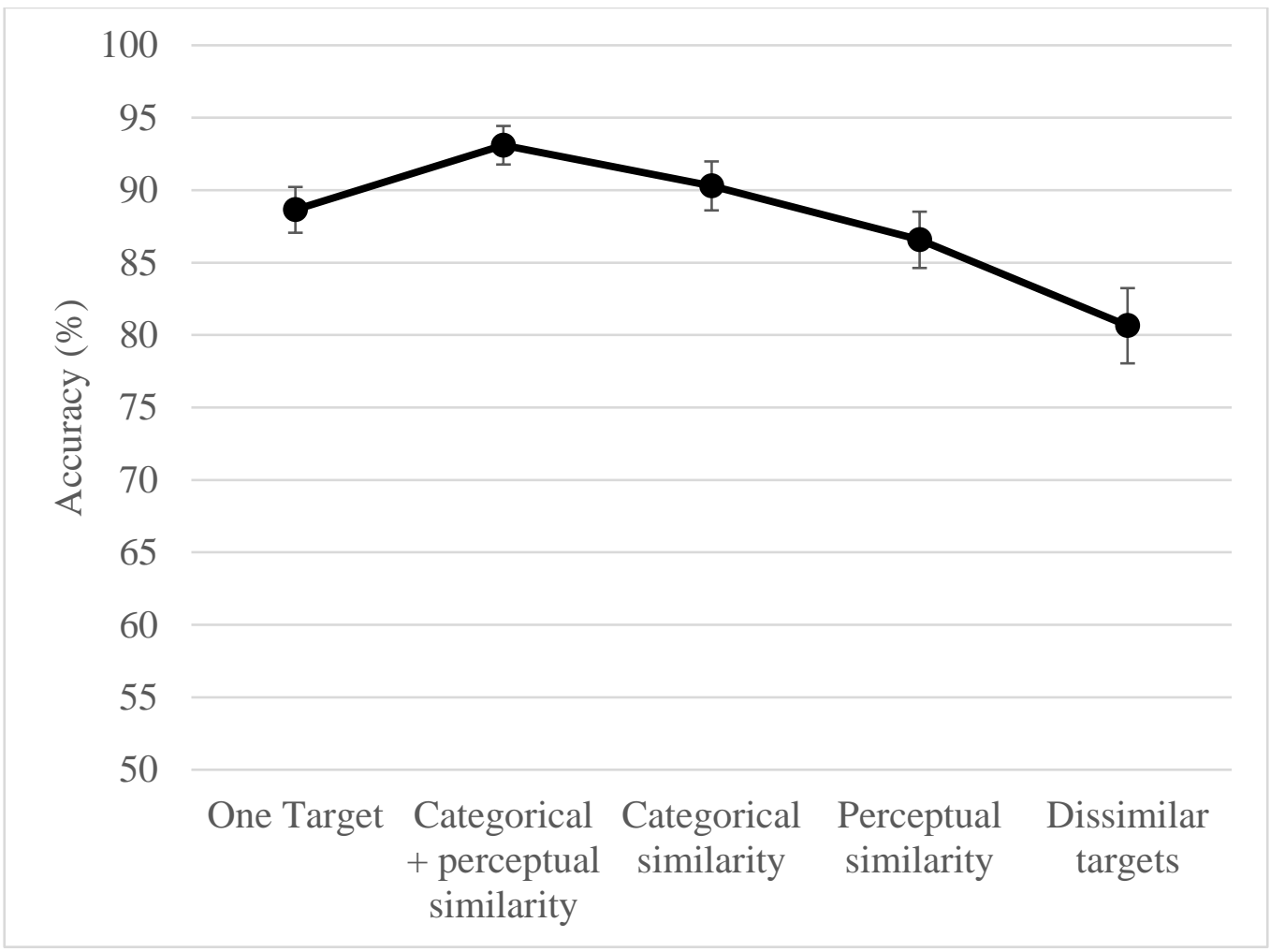

Figure 2. Results of Experiment 1 (accuracy). Error bars represent standard error means

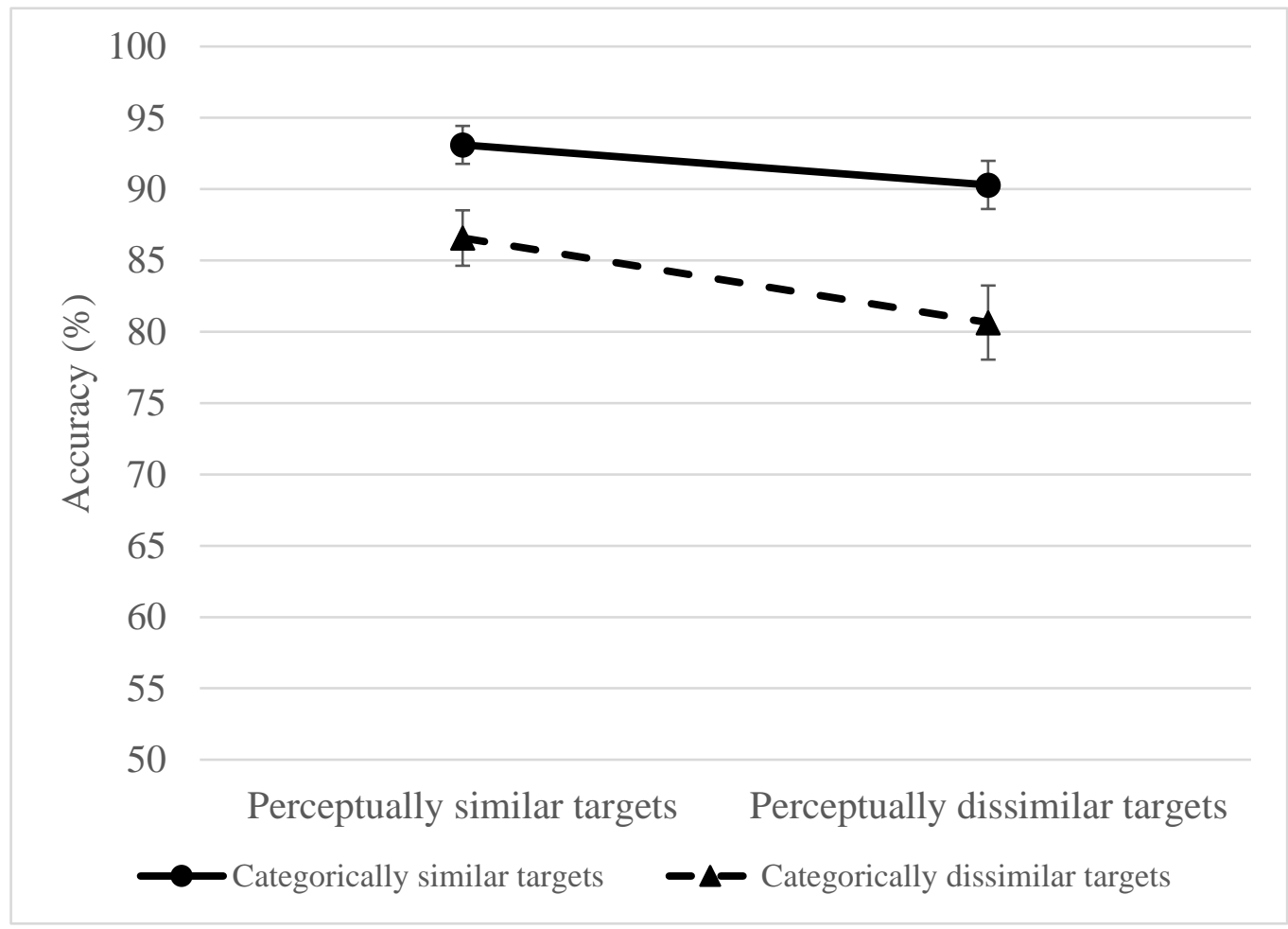

Figure 3. Results of Experiment 1 (accuracy). Error bars represent standard error means 
Table 1. Experiment 1, results of pairwise comparisons for accuracy, significant pvalues are in bold

\begin{tabular}{|c|c|c|}
\hline Condition & Condition & p-Value \\
\hline \multirow{15}{*}{2 targets, identical } & 2 targets, identical & .003 \\
\hline & 2 targets, categorical & \\
\hline & but not perceptual similarity & .702 \\
\hline & 2 targets, perceptual & \\
\hline & but not categorical similarity & 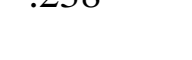 \\
\hline & 2 targets, no & \\
\hline & categorical or perceptual & \\
\hline & similarity & .004 \\
\hline & 2 targets, categorical & \\
\hline & but not perceptual similarity & .279 \\
\hline & 2 targets, perceptual & \\
\hline & but not categorical similarity & .002 \\
\hline & 2 targets, no & \\
\hline & categorical or perceptual & \\
\hline & similarity & $<.001$ \\
\hline \multirow{6}{*}{$\begin{array}{l}2 \text { targets, categorical } \\
\text { but not perceptual similarity }\end{array}$} & 2 targets, perceptual & 00 \\
\hline & but not categorical similarity & \\
\hline & & \\
\hline & 2 targets, no & \\
\hline & categorical or perceptual & \\
\hline & similarity & .002 \\
\hline \multirow{3}{*}{$\begin{array}{l}2 \text { targets, perceptual } \\
\text { but not categorical similarity }\end{array}$} & 2 targets, no & \\
\hline & categorical or perceptual & \\
\hline & similarity & .003 \\
\hline
\end{tabular}




\section{Reaction time (first click)}

One-way repeated measures ANOVA revealed the significant impact of target similarity factor: $F(4,108)=33.920 ; p<.001 ; \eta p^{2}=.557$. Pairwise comparisons with Holm-Bonferroni adjustment revealed significant differences between following conditions: one target and all other conditions, two targets with categorical similarity and two targets with no similarity, two targets with perceptual similarity and two targets with no similarity. The results are presented in Fig.4 and Table 2.

Two-way repeated measures ANOVA revealed no significant effect of perceptual similarity factor, $F(1,27)=2.628 ; p=.117 ; \eta p^{2}=.089$. The effect of categorical similarity was also not significant, $F(1,27)=1.934 ; p=.176 ; \eta p^{2}=.067$. The interaction was significant, $F$ $(1,27)=14.942 ; p=.001 ; \eta p^{2}=.356$.

Due to significant interaction, separate rmANOVAs were made for perceptually similar and dissimilar targets (the factor was categorical similarity), as well as separate rmANOVAs were made for categorically similar and dissimilar targets (the factor was perceptual similarity). For perceptually similar targets, the effect of categorical similarity was not significant, $F(1,27)=$ $3.056 ; p=.092 ; \eta p^{2}=.102$. For perceptually dissimilar targets, the effect of categorical similarity was significant, $F(1,27)=7.820 ; p=.009 ; \eta p^{2}=.225$. For categorically similar targets, the effect of perceptual similarity was not significant, $F(1,27)=2.200 ; p=.150 ; \eta p^{2}=.075$. For categorically dissimilar targets, the effect of perceptual similarity was significant, $F(1,27)=$ $12.861 ; p=.001 ; \eta p^{2}=.323$.

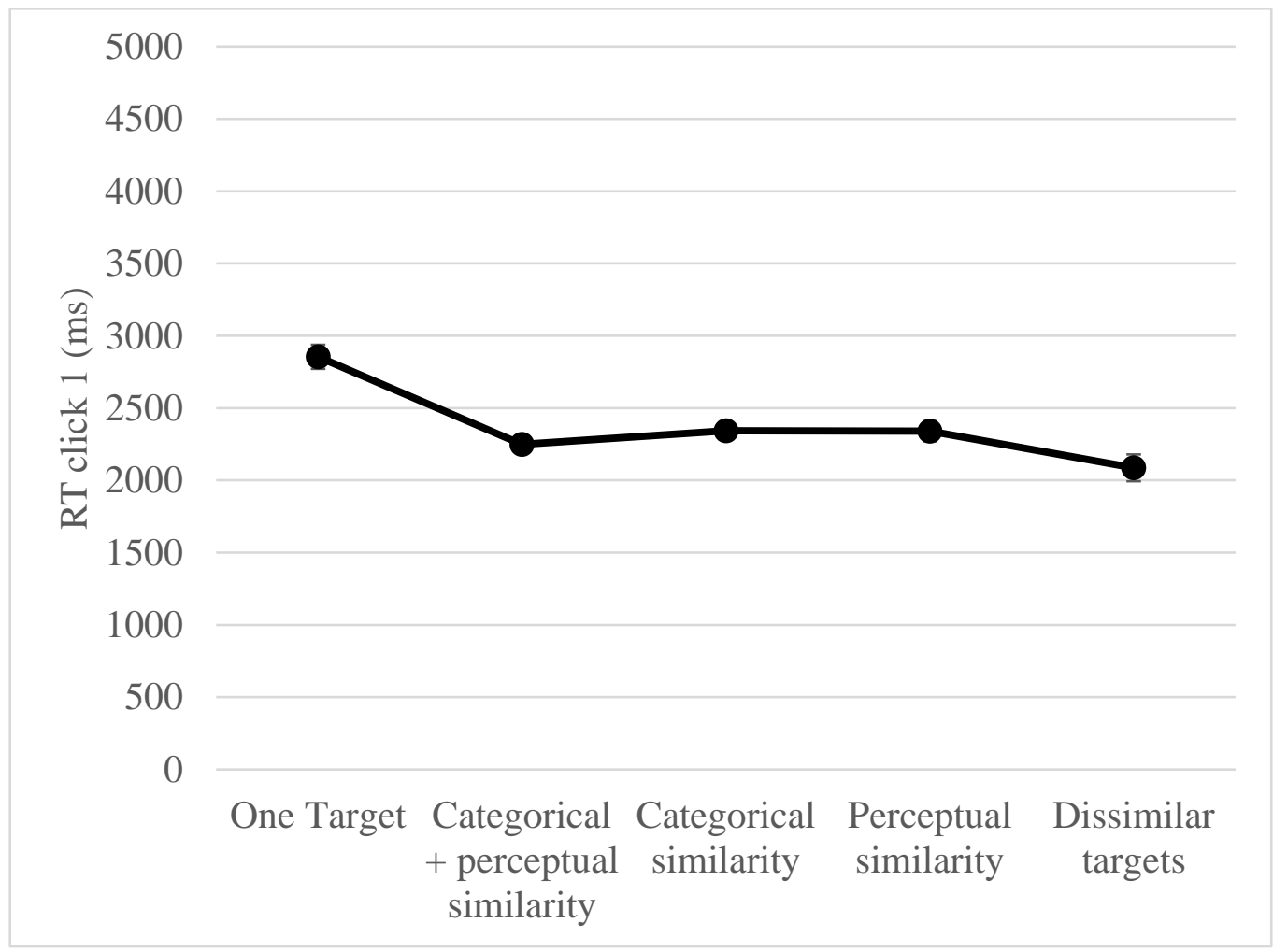

Figure 4. Results of Experiment 1 (RT, first mouse click). Error bars represent standard error means 
Table 2. Experiment 1, results of pairwise comparisons for reaction time (first click), significant $\mathrm{p}$-values are in bold

\begin{tabular}{|c|c|c|}
\hline Condition & Condition & p-Value \\
\hline \multirow[t]{8}{*}{1 target } & 2 targets, identical & $<.001$ \\
\hline & 2 targets, categorical & \\
\hline & but not perceptual similarity & $<.001$ \\
\hline & 2 targets, perceptual & \\
\hline & but not categorical similarity & $<.001$ \\
\hline & 2 targets, no & \\
\hline & categorical or perceptual & \\
\hline & similarity & $<.001$ \\
\hline \multirow[t]{7}{*}{2 targets, identical } & 2 targets, categorical & \\
\hline & but not perceptual similarity & 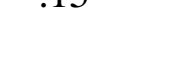 \\
\hline & 2 targets, perceptual & 097 \\
\hline & but not categorical similarity & \\
\hline & 2 targets, no & \\
\hline & categorical or perceptual & \\
\hline & similarity & .281 \\
\hline \multirow{5}{*}{$\begin{array}{l}2 \text { targets, categorical } \\
\text { but not perceptual similarity }\end{array}$} & 2 targets, perceptual & 974 \\
\hline & but not categorical similarity & \\
\hline & 2 targets, no & \\
\hline & categorical or perceptual & \\
\hline & similarity & .047 \\
\hline \multirow{3}{*}{$\begin{array}{l}2 \text { targets, perceptual } \\
\text { but not categorical similarity }\end{array}$} & 2 targets, no & \\
\hline & categorical or perceptual & \\
\hline & similarity & .008 \\
\hline
\end{tabular}

\section{Reaction time (second click)}

One-way repeated measures ANOVA revealed the significant impact of target similarity factor: $F(1,34)=47.606 ; p<.001 ; \eta p^{2}=.638$. Pairwise comparisons with Holm-Bonferroni adjustment revealed significant differences between the following conditions: one target and all 
other conditions, two identical targets and two targets with categorical similarity, two identical targets and two targets with no similarity, two targets with categorical similarity and two targets with perceptual similarity, two targets with categorical similarity and two targets with no similarity. The results are presented in Fig.5 and Table 3.

Two-way repeated measures ANOVA revealed no significant effect of perceptual similarity factor, $F(1,27)=0.948 ; p=.339 ; \eta p^{2}=.034$. The effect of categorical similarity was significant, $F(1,27)=27.901 ; p<.001 ; \eta p^{2}=.508$. The interaction was significant, $F(1,27)=$ 5.148; $p=.031 ; \eta p^{2}=.161$.

Due to significant interaction, separate rmANOVAs were made for perceptually similar and dissimilar targets (the factor was categorical similarity), as well as separate rmANOVAs were made for categorically similar and dissimilar targets (the factor was perceptual similarity). For perceptually similar targets, the effect of categorical similarity was significant, $F(1,27)=$ 5.704; $p=.024 ; \eta p^{2}=.174$. For perceptually dissimilar targets, the effect of categorical similarity was also significant, $F(1,27)=48.443 ; p<.001 ; \eta p^{2}=.642$. For categorically similar targets, the effect of perceptual similarity was significant, $F(1,27)=5.069 ; p=.033 ; \eta p^{2}=.158$. For categorically dissimilar targets, the effect of perceptual similarity was not significant, $F(1,27)=$ $1.099 ; p=.304 ; \eta p^{2}=.039$.

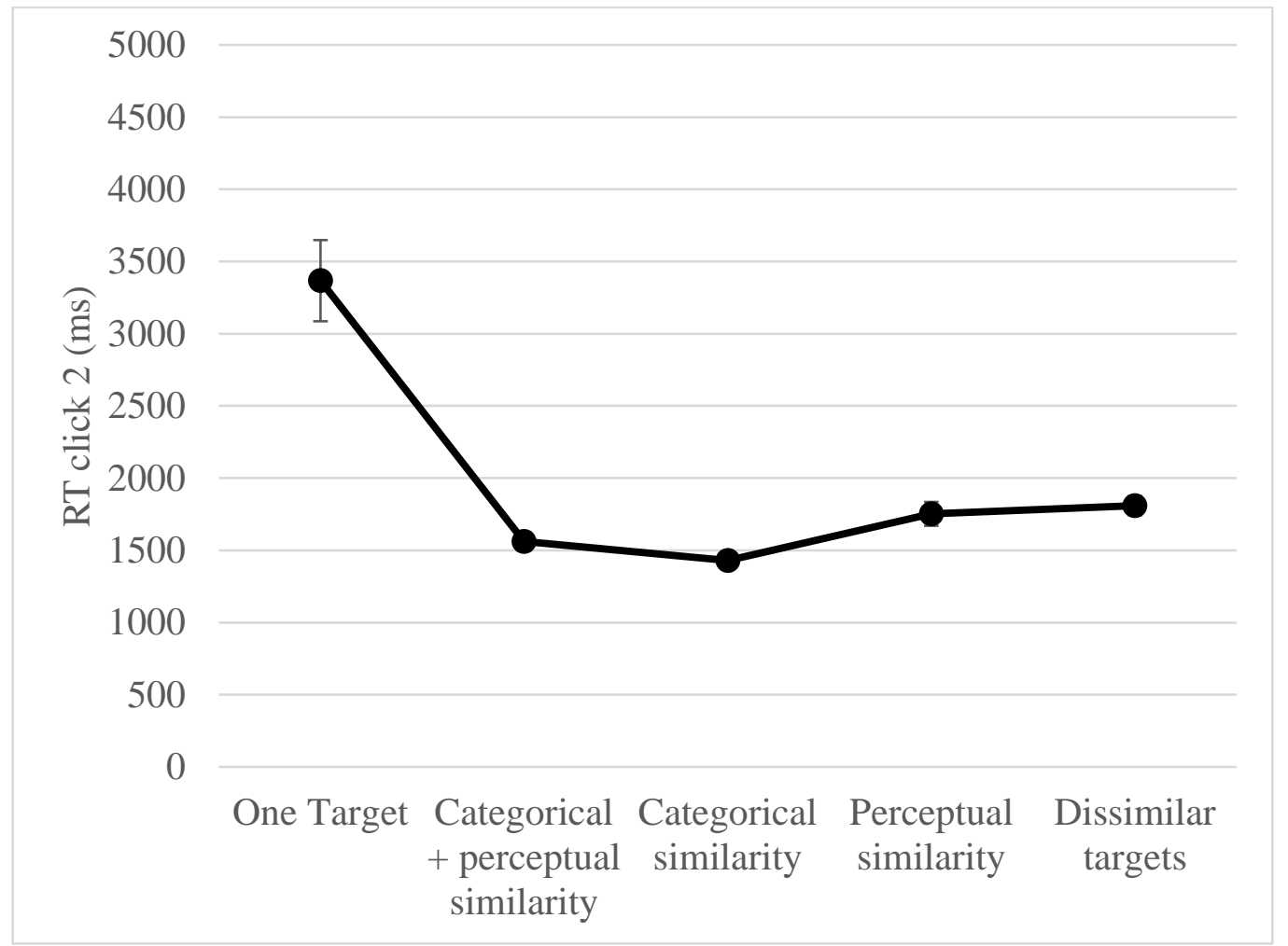

Figure 5. Results of Experiment 1 (RT, second mouse click). Error bars represent standard error means 
Table 3. Experiment 1, results of pairwise comparisons for reaction time (second click), significant $p$-values are in bold

\begin{tabular}{|c|c|c|}
\hline Condition & Condition & $\mathrm{p}$-Value \\
\hline \multirow[t]{8}{*}{1 target } & 2 targets, identical & $<.001$ \\
\hline & 2 targets, categorical & \\
\hline & but not perceptual similarity & $<.001$ \\
\hline & 2 targets, perceptual & \\
\hline & but not categorical similarity & $<.001$ \\
\hline & 2 targets, no & \\
\hline & categorical or perceptual & \\
\hline & similarity & $<.001$ \\
\hline \multirow[t]{7}{*}{2 targets, identical } & 2 targets, categorical & \\
\hline & but not perceptual similarity & . USנ \\
\hline & 2 targets, perceptual & \\
\hline & but not categorical similarity & .073 \\
\hline & 2 targets, no & \\
\hline & categorical or perceptual & \\
\hline & similarity & .004 \\
\hline \multirow{5}{*}{$\begin{array}{l}2 \text { targets, categorical } \\
\text { but not perceptual similarity }\end{array}$} & 2 targets, perceptual & \\
\hline & but not categorical similarity & $<.001$ \\
\hline & 2 targets, no & \\
\hline & categorical or perceptual & \\
\hline & similarity & $<.001$ \\
\hline 2 targets, perceptual & 2 targets, no & \\
\hline but not categorical similarity & $\begin{array}{c}\text { categorical or perceptual } \\
\text { similarity }\end{array}$ & .304 \\
\hline
\end{tabular}

\section{Discussion}

SSM errors were found only for the condition with two targets that shared neither categorical, nor perceptual similarity. This provides evidence for both perceptual and categorical set hypotheses. As expected, the accuracy decreased with the reduction of target similarity. In all 
conditions, when targets shared some degree of similarity, the percentage of correct answers was significantly higher than in case of absence of similarity of targets. The significant difference between conditions with two targets that shared only categorical or only perceptual similarity was not revealed, and two-way ANOVA revealed the impact of both perceptual and categorical similarity factors, which makes it difficult to discuss the possible superiority of the categorical factor in this case.

In cases of identical or categorically similar targets the second letter was found significantly faster than in case of different targets. When targets were perceptually but not categorically similar, the participants tended to identify second targets not faster than in no similarity condition. What is more, categorically similar second target tended to be found significantly faster than the perceptually similar one. Altogether, that suggests not only the influence of target similarity, but also the superiority of categorical set in SSM, when it comes to the speed of finding the second target.

What is interesting, is the fact that in case of two identical targets the accuracy was significantly higher than in case of one target. That might be explained by possible "pop-out" effect for the condition of two identical targets. In the condition with only one target it had low salience, while in the condition with identical targets the less visible letter had to be found the second in order for the trial to be counted as a correct one. However, "pop-out" effects are typically only observed for visually salient stimuli, and the second target was always less salient than the first one. Another explanation is that detecting a first target primed the detection of identical targets (e.g., Maljkovic \& Nakayama, 1994, 1996). Thus, it seems possible that in the condition with identical letters the identification of the first one very successfully guided attention towards the perceptually same item, which resulted in the exceeding of accuracy. However, another possible explanation is that probably not all 50 trials in case of conditions with two targets were analyzed due to the specifics of data analysis in this experimental design. Even if the participant found both targets but the firstly identified letter had low salience, such trials were not analyzed, which could lead, to some extent, to inaccuracy in data representation. This could also explain other unexpected findings in this experiment, for instance, the difference in reaction time for the first mouse click between conditions with two targets with perceptual similarity and no similarity. In this case, the significantly slower search for only one targets reflects the classic dynamics shown in previous research (Kwak et al, 1991). The more targets there are presented on screen, the less time it takes to find at least one of them. The differences between any other conditions, however, were expected to be insignificant. Once again, the difference between two last condition seems to be the result of the analysis method. For the second mouse click, the time it took to become certain in the absence of the second target, was 
significantly higher in comparison with all other conditions. This is not a surprise, because it takes more time to search through the whole screen and be sure that the target was not missed. There are a few critical points in regards to the first experiment. Firstly, the ceiling effect was achieved judging by the overall high results of the participants. That could have evened out some effects, that might be more distinct in more difficult tasks. Another important point is that different letters presented in the same case are not necessarily more perceptually similar than different letters presented in different cases. Moreover, it is quite possible that upper-case and lower-case letters can be perceived as belonging to different categories. That means that in conditions where only one type of similarity is presented, the possible interference might have occurred. Thus, no significant differences in accuracy between those two conditions could reflect the disadvantages of experimental design. Finally, one last factor could contribute to this very issue as well. The letters varied in shades of gray, which was made in order to make darker letters more perceptually noticeable than lighter ones. In case of two targets one of them was always dark gray, while the other one was light gray. Therefore, in all conditions with two targets the letters could be perceived as those of different colors, while color is known to be one of the strongest perceptual factors (Zhuang \& Papathomas, 2011).

In order to avoid the confounds described above, it was decided to conduct a second experiment with an improved experimental design.

\section{Experiment 2}

\section{Method}

\section{Participants}

The participants took part in the experiment for the class credit, all students from National Research University Higher School of Economics, with normal or corrected to normal vision, without any neurological or psychological problems and native Russian speakers. None of them took part in the first experiment. Results from 1 participant were excluded due to misunderstanding the instruction, so all in all data from 34 subjects were analyzed. Participants were 4 males and 30 females, and their age varied from 18 to $23(\mathrm{M}=20.05, \mathrm{SD}=0.89)$.

\section{Stimuli}

The stimuli material and apparatus were the same as in the experiment 1 . The only exception was that the color of the letters was varied instead of the way of writing in order to determine the perceptual similarity. The letters could be black (CIE: .345, .358, 0) or white (CIE: .313, .329, 100.0). All the letters were upper-case. Thus, the categorical similarity of targets was determined by the letter identity, while the perceptual similarity was determined by the color. Overall, 6 experimental conditions were created: one target 
(black or white), no targets, two identical targets, two targets with categorical similarity, two targets with perceptual similarity, two targets with no similarity. The rest was the same as in experiment 1. Example of a trial is presented in Fig.6.

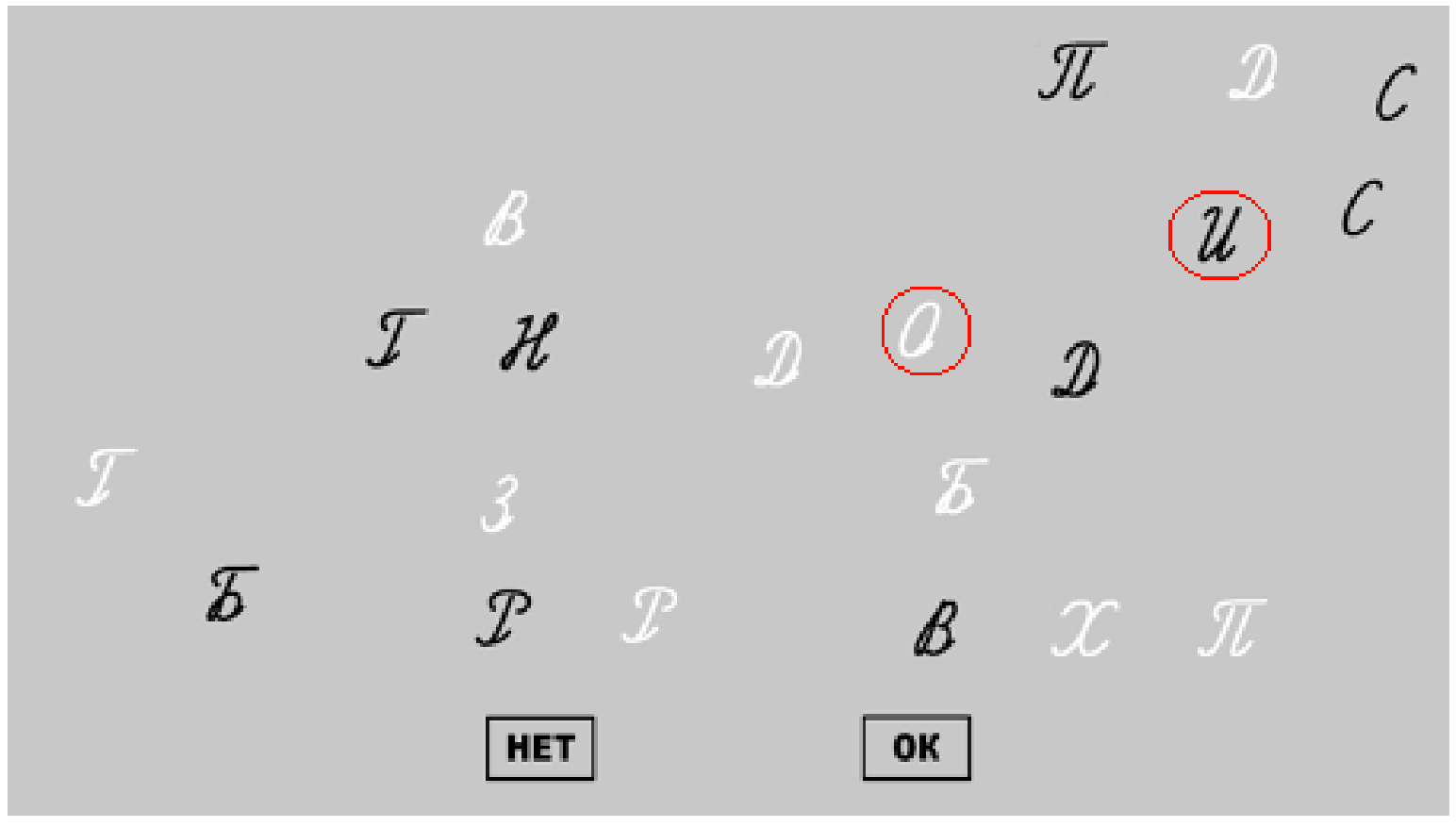

Figure 6. An example of experimental trial in Experiment 2 (condition for two targets, with neither perceptual nor categorical similarity - targets are marked with red circles)

\section{Procedure}

Procedure was exactly the same as described in experiment 1 . Overall, there were 300 experimental trials, 50 for each condition. The training session consisted of 12 trials.

\section{Results}

Data analysis was similar to Experiment 1. Accuracy and reaction time for conditions with two targets (with four different degrees of target similarity) and one target were analyzed. Condition with no targets did was not included in the analysis. The analysis was made using SPSS 20.0. Repeated measures ANOVA and pairwise comparisons with Holm-Bonferroni adjustment were used. The Greenhouse-Geisser corrections were applied, when Mauchly's sphericity tests were significant.

For dual-target trials, the percentage of correct responses was calculated only for those trials, when at least one target was found (for an example of this kind of analysis, see e.g. Gorbunova, 2017). Due to the absence of saliency manipulation, it did not matter, what target was found first.

\section{Accuracy}

One-way repeated measures ANOVA revealed the significant impact of target similarity factor: $F(3,83)=10,599 ; p<.001 ; \eta p^{2}=.243$. Pairwise comparisons with Holm-Bonferroni adjustment revealed significant differences between following conditions: one target and two 
targets with perceptual similarity, one target and two targets with no similarity, two identical targets and two targets with perceptual similarity, two identical targets and two targets with no similarity, two targets with categorical similarity and two targets with no similarity. The results are presented in Fig.7-8 and Table 4.

Two-way repeated measures ANOVA revealed no significant effect of perceptual similarity factor, $F(1,33)=1.628 ; p=.211 ; \eta p^{2}=.047$. The effect of categorical similarity was significant, $F(1,33)=21.406 ; p<.001 ; \eta p^{2}=.393$. The interaction was not significant, $F(1,33)$ $=1.229 ; p=.276 ; \eta p^{2}=.036$.

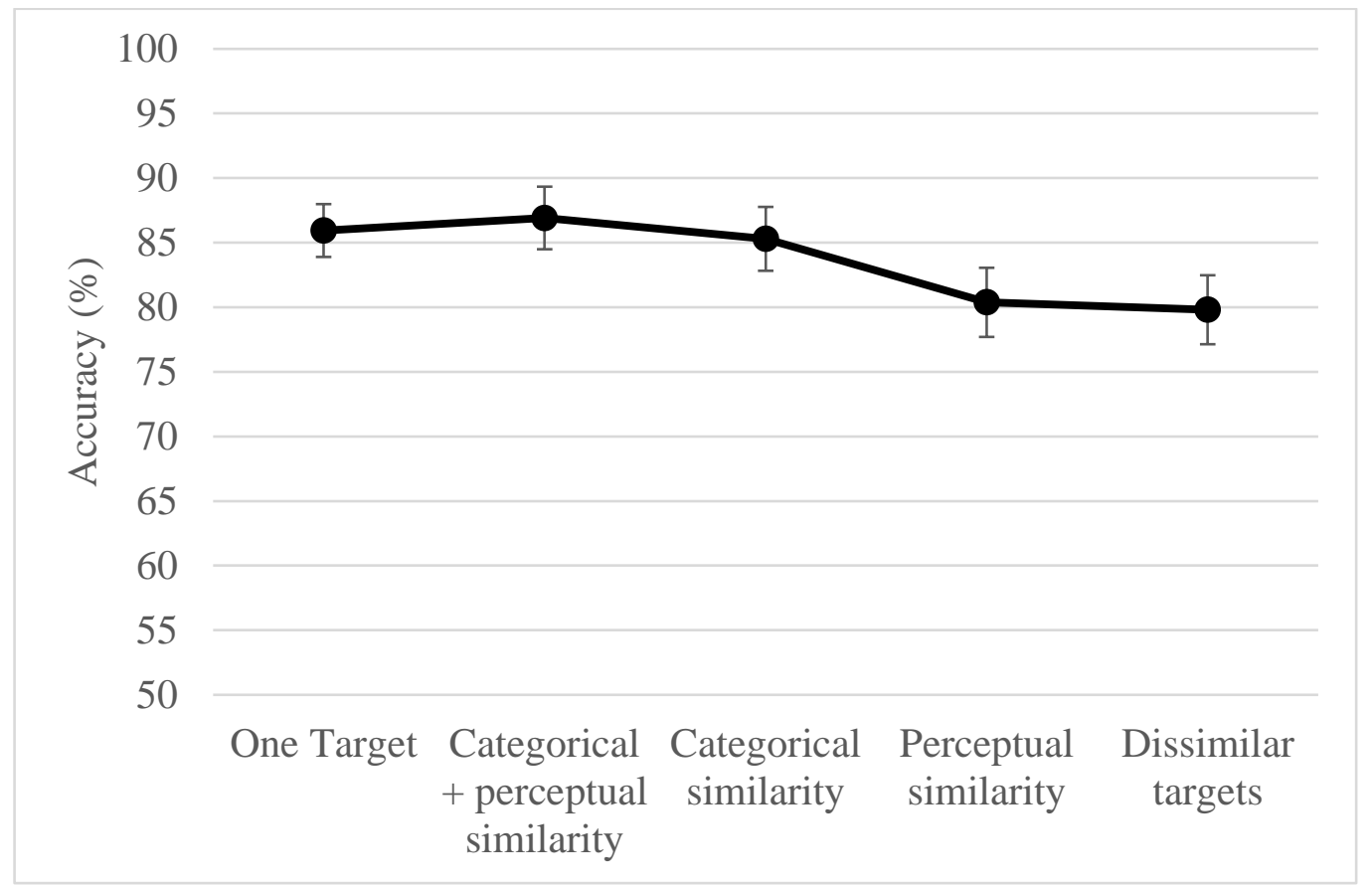

Figure 7. Results of Experiment 2 (accuracy). Error bars represent standard error means

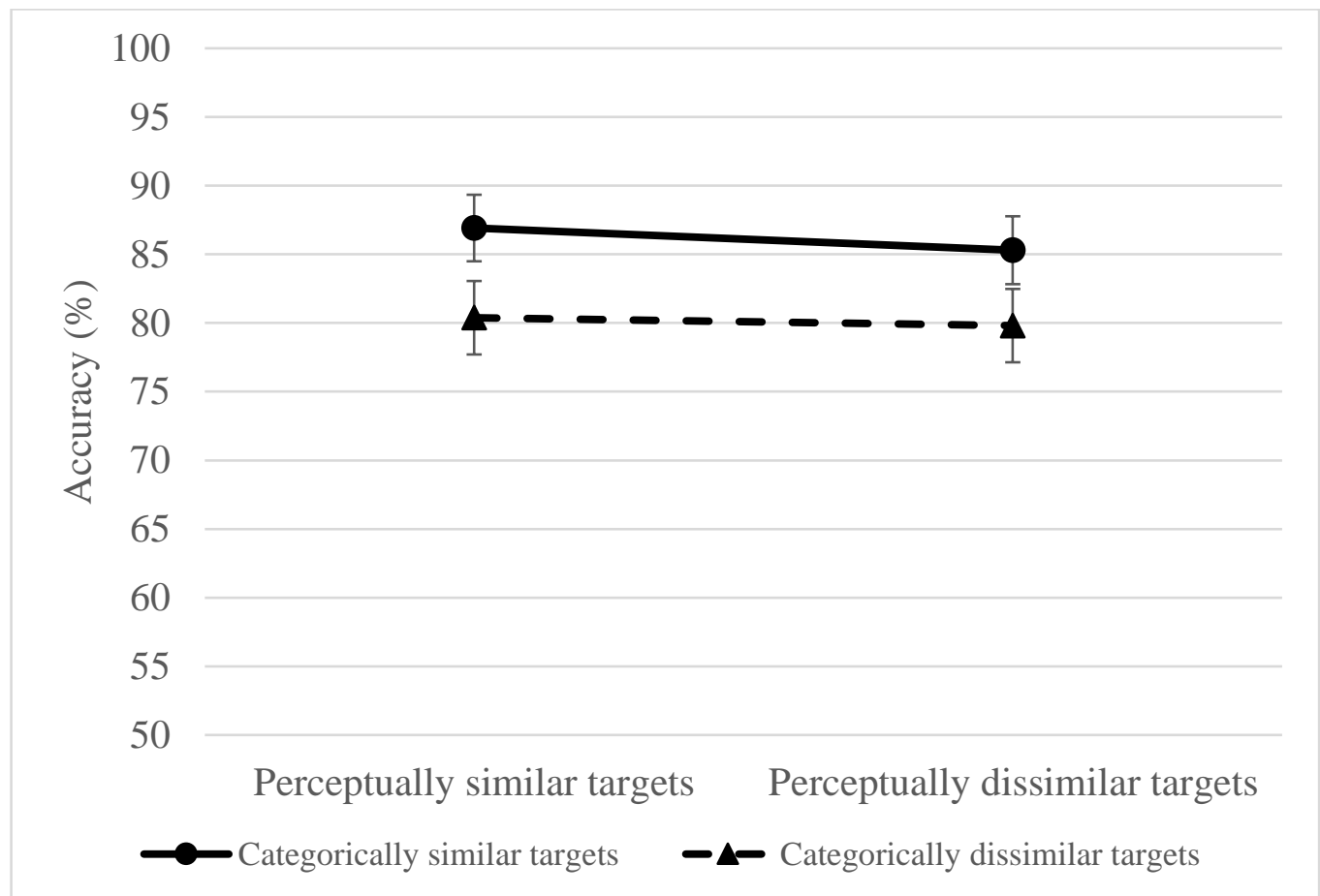

Figure 8. Results of Experiment 2 (accuracy). Error bars represent standard error means 
Table 4. Experiment 2, results of pairwise comparisons for accuracy, significant p-values are in bold

\begin{tabular}{|c|c|c|}
\hline Condition & Condition & $\mathrm{p}$-Value \\
\hline \multirow[t]{8}{*}{1 target } & 2 targets, identical & .552 \\
\hline & 2 targets, categorical & \multirow{3}{*}{.727} \\
\hline & but not perceptual similarity & \\
\hline & 2 targets, perceptual & \\
\hline & but not categorical similarity & \multirow[t]{3}{*}{.003} \\
\hline & 2 targets, no & \\
\hline & categorical or perceptual & \\
\hline & similarity & .002 \\
\hline \multirow[t]{7}{*}{2 targets, identical } & 2 targets, categorical & \multirow{3}{*}{.111} \\
\hline & but not perceptual similarity & \\
\hline & 2 targets, perceptual & \\
\hline & but not categorical similarity & \multirow[t]{3}{*}{.0008} \\
\hline & 2 targets, no & \\
\hline & categorical or perceptual & \\
\hline & similarity & \multirow[t]{2}{*}{$<.001$} \\
\hline 2 targets, categorical & 2 targets, perceptual & \\
\hline \multirow[t]{4}{*}{ but not perceptual similarity } & but not categorical similarity & \multirow[t]{3}{*}{.067} \\
\hline & 2 targets, no & \\
\hline & categorical or perceptual & \\
\hline & similarity & \multirow[t]{2}{*}{.001} \\
\hline 2 targets, perceptual & 2 targets, no & \\
\hline but not categorical similarity & categorical or perceptual & \multirow[t]{2}{*}{.559} \\
\hline & similarity & \\
\hline
\end{tabular}




\section{Reaction time (first click)}

One-way repeated measures ANOVA revealed the significant impact of target similarity factor: $F(3,92)=62,510 ; p<.001 ; \eta p^{2}=.654$. Pairwise comparisons with Holm-Bonferroni adjustment revealed significant differences between following conditions: one target and all other conditions, two identical targets and two targets with categorical similarity, two identical targets and two targets with perceptual similarity. The results are presented in Fig.9 and Table 5.

Two-way repeated measures ANOVA revealed no significant effect of perceptual similarity factor, $F(1,33)=1.452 ; p=.237 ; \eta p^{2}=.042$. The effect of categorical similarity was also not significant, $F(1,33)=1.537 ; p=.224 ; \eta p^{2}=.044$. The interaction was significant, $F$ $(1,33)=12.327 ; p=.001 ; \eta p^{2}=.272$.

Due to significant interaction, separate rmANOVAs were made for perceptually similar and dissimilar targets (the factor was categorical similarity), as well as separate rmANOVAs were made for categorically similar and dissimilar targets (the factor was perceptual similarity). For perceptually similar targets, the effect of categorical similarity was significant, $F(1,33)=$ $8.227 ; p=.007 ; \eta p^{2}=.200$. For perceptually dissimilar targets, the effect of categorical similarity was not significant, $F(1,33)=1.890 ; p=.178 ; \eta p^{2}=.054$. For categorically similar targets, the effect of perceptual similarity was significant, $F(1,33)=9.176 ; p=.005 ; \eta p^{2}=.218$. For categorically dissimilar targets, the effect of perceptual similarity was not significant, $F(1,33)=$ $1.809 ; p=.188 ; \eta p^{2}=.052$.

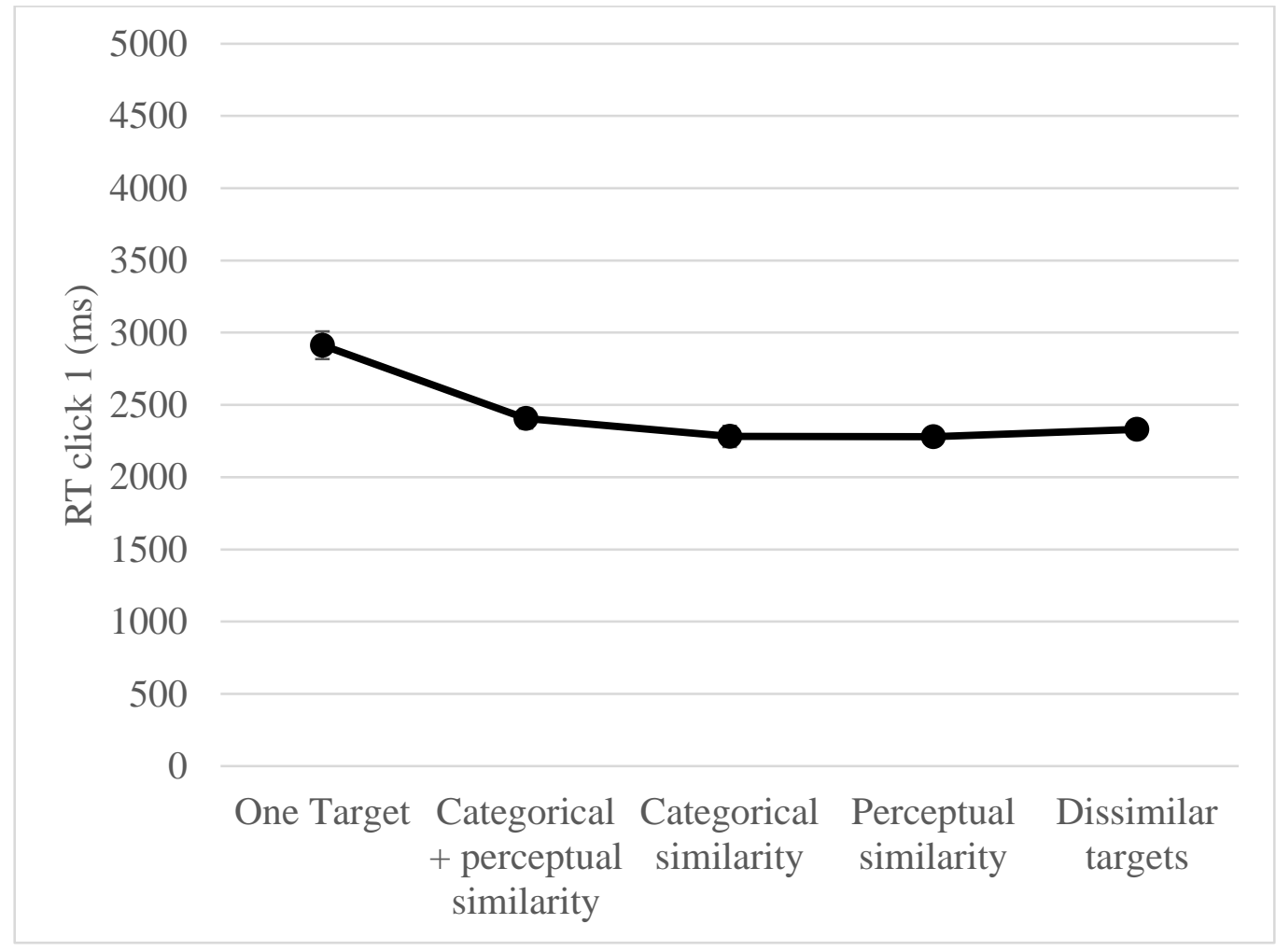

Figure 9. Results of Experiment 2 (RT, first mouse click). Error bars represent standard error means 
Table 5. Experiment 2, results of pairwise comparisons for reaction time (first click), significant $p$-values are in bold

\begin{tabular}{|c|c|c|}
\hline Condition & Condition & p-Value \\
\hline \multirow[t]{8}{*}{1 target } & 2 targets, identical & $<.001$ \\
\hline & 2 targets, categorical & $<.001$ \\
\hline & but not perceptual similarity & \\
\hline & 2 targets, perceptual & $<.001$ \\
\hline & but not categorical similarity & \\
\hline & 2 targets, no & \multirow{3}{*}{$<.001$} \\
\hline & categorical or perceptual & \\
\hline & similarity & \\
\hline \multirow[t]{7}{*}{2 targets, identical } & 2 targets, categorical & \multirow{2}{*}{.028} \\
\hline & but not perceptual similarity & \\
\hline & 2 targets, perceptual & \multirow{2}{*}{.036} \\
\hline & but not categorical similarity & \\
\hline & 2 targets, no & \\
\hline & categorical or perceptual & .24 \\
\hline & similarity & \\
\hline \multirow{5}{*}{$\begin{array}{l}2 \text { targets, categorical } \\
\text { but not perceptual similarity }\end{array}$} & 2 targets, perceptual & \multirow{2}{*}{.968} \\
\hline & but not categorical similarity & \\
\hline & 2 targets, no & \multirow{3}{*}{.178} \\
\hline & categorical or perceptual & \\
\hline & similarity & \\
\hline 2 targets, perceptual & 2 targets, no & \multirow{3}{*}{.188} \\
\hline but not categorical similarity & categorical or perceptual & \\
\hline & similarity & \\
\hline
\end{tabular}

\section{Reaction time (second click)}

One-way repeated measures ANOVA revealed the significant impact of target similarity factor: $F(2,51)=94,320 ; p<.001 ; \eta p^{2}=.741$. Pairwise comparisons with Holm-Bonferroni adjustment revealed significant differences between following conditions: one target and all 
other conditions, two identical targets and two targets with perceptual similarity, two identical targets and two targets with no similarity, two targets with categorical similarity and two targets with perceptual similarity, two targets with categorical similarity and two targets with no similarity. The results are presented in Fig.10 and Table 6.

Two-way repeated measures ANOVA revealed no significant effect of perceptual similarity factor, $F(1,33)=1.978 ; p=.169 ; \eta p^{2}=.057$. The effect of categorical similarity was significant, $F(1,33)=73.227 ; p<.001 ; \eta p^{2}=.689$. The interaction was not significant, $F(1,33)$ $=0.898 ; p=.350 ; \eta p^{2}=.027$.

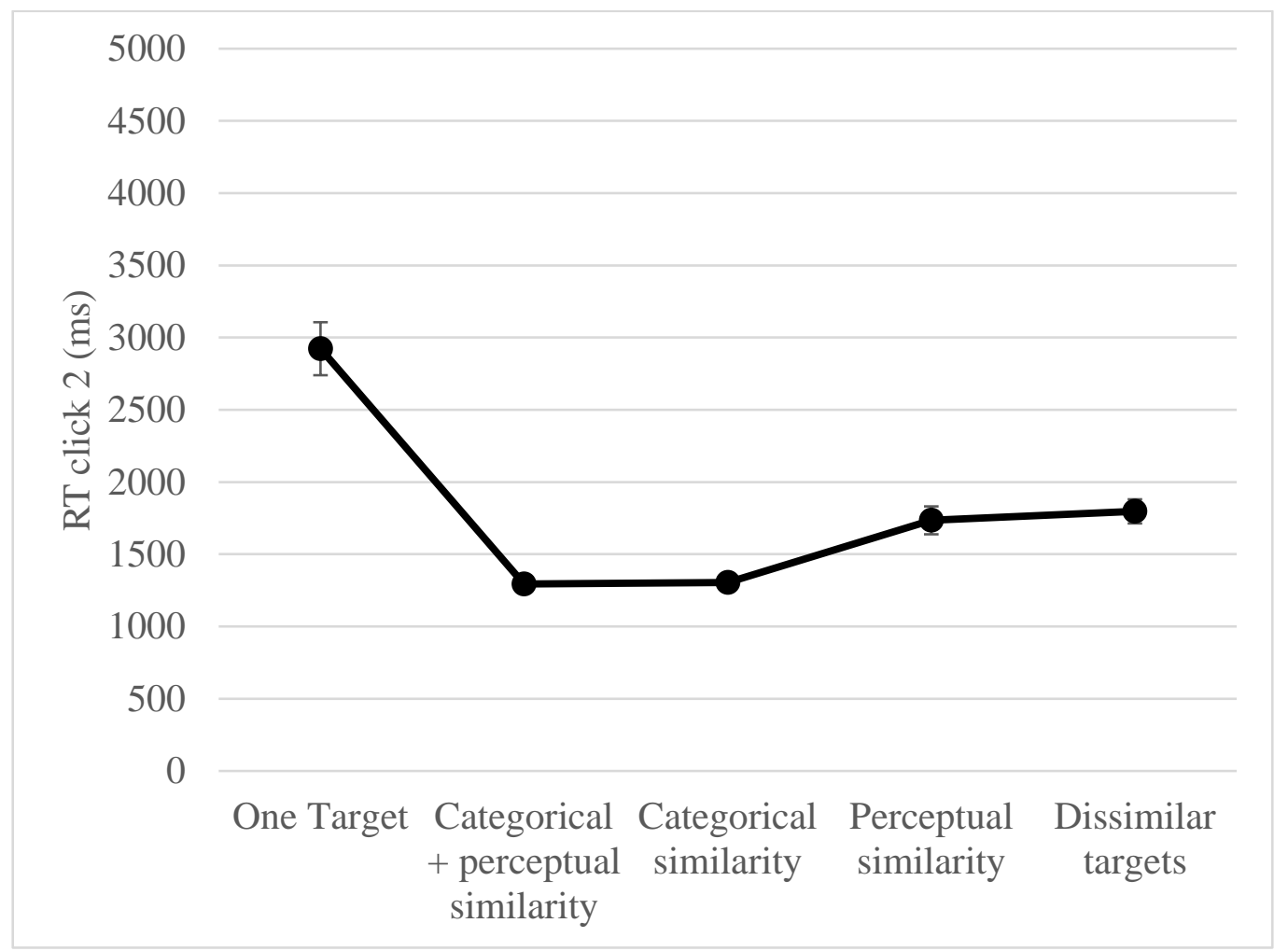

Figure 10. Results of Experiment 2 (RT, second mouse click). Error bars represent standard error means 
Table 6. Experiment 2, results of pairwise comparisons for reaction time (second click), significant p-values are in bold

\begin{tabular}{|c|c|c|}
\hline Condition & Condition & p-Value \\
\hline \multirow[t]{8}{*}{1 target } & 2 targets, identical & $<.001$ \\
\hline & 2 targets, categorical & $<.001$ \\
\hline & but not perceptual similarity & \\
\hline & 2 targets, perceptual & $<.001$ \\
\hline & but not categorical similarity & \\
\hline & 2 targets, no & $<.001$ \\
\hline & categorical or perceptual & \\
\hline & similarity & \\
\hline \multirow[t]{7}{*}{2 targets, identical } & 2 targets, categorical & 676 \\
\hline & but not perceptual similarity & \\
\hline & 2 targets, perceptual & \\
\hline & but not categorical similarity & $<.001$ \\
\hline & 2 targets, no & \\
\hline & categorical or perceptual & \\
\hline & similarity & $<.001$ \\
\hline 2 targets, categorical & 2 targets, perceptual & \\
\hline \multirow[t]{4}{*}{ but not perceptual similarity } & but not categorical similarity & $<.001$ \\
\hline & 2 targets, no & \\
\hline & categorical or perceptual & \\
\hline & similarity & $<.001$ \\
\hline \multirow{3}{*}{$\begin{array}{l}2 \text { targets, perceptual } \\
\text { but not categorical similarity }\end{array}$} & 2 targets, no & \\
\hline & categorical or perceptual & \\
\hline & similarity & .357 \\
\hline
\end{tabular}

\section{Discussion}

SSM were observed only for conditions with two perceptually but not categorically similar targets and for two different targets. In the conditions with two identical and categorically but not perceptually similar targets the accuracy was significantly higher than in the condition 
with two different targets. There were no significant differences between conditions with two just categorically and two just perceptually similar targets. These results support the hypothesis that the more similarities, either categorical or perceptual, the two targets share, the higher the accuracy of detecting the second letter is. These results correspond to previously found data on targets similarity role in SSM (Gorbunova 2017, Lanina \& Gorbunova, 2018). The reduction of accuracy in this case can be explained by both perceptual and categorical biases towards recently found targets and the lack of attentional resources or those of working memory (however, the latter account should be directly tested in the further research). It would also be quite rational to take both of these explanations into consideration, as they do not contradict each other.

Despite the fact that there were no significant differences revealed between condition with just categorical and just perceptual difference, two-way ANOVA revealed no significant effect of perceptual similarity factor, whereas the effect of categorical similarity was significant. At this point, it is possible to make an assumption that categorical superiority might take place. It is reasonable, since the perceptual similarity of targets still did not help to avoid the emergence of SSM, which can be seen from the results.

The analysis of reaction time for the second mouse click revealed the shortest second target detection RTs for the conditions with two identical and two categorically but not perceptually similar targets. What is more important, it took significantly more time to find the second target if it was just perceptually similar to the first one, unlike the condition with only categorical similarity of targets. Two-way ANOVA for the second mouse click also revealed the effect of categorical, but not perceptual similarity. That means that the superiority of categorical set was shown in this experiment. It can be discussed in the light of Biggs and colleagues' results of their big data study (Biggs et al 2015). It could be argued that the current experiment had a manipulated experimental control of independent variables, which enabled to separate the influence of categorical and perceptual similarity. In the Airport Scanner study objects from real world were used. This is a great advantage of that research, due to its ecological validity. At the same time, on the whole, real-world objects do have many additional features that always interfere with the ones that are used as independent variables in experimental studies. By using less perceptually complex stimuli we were able to achieve the desired separation of both categorical and perceptual factors and investigate their influence apart from each other, as well as their combined role in SSM.

However, many of the present findings were observed on response time rather than accuracy. It is possible to suggest that the present findings reflect more general dual-target search costs rather than SSM errors (e.g., Menneer, Cave, \& Donnelly, 2009; Menneer, Donnelly, Godwin, \& Cave, 2010; Stroud, Cave, Menner, \& Donnelly, 2012). SSM errors were not observed in many of the two-target conditions. This differs from many SSM studies, in which 
SSM rates are typically much higher. One possible explanation for these findings concerns the relative proportion of two-target trials. Because these trials occurred twice as often as one-target trials, participants may have been biased to search for a second target on each trial. Indeed, previous evidence suggests that the relative proportion of two-target trials can strongly influence SSM rates (e.g., Fleck et al., 2010).

Moreover, the instructions in this experiment were to find the vowels. At that point, the letter identity was task relevant feature, while the brightness was task-irrelevant, which challenges the categorical similarity superiority. At that point, a third experiment was conducted. In this experiment, both perceptual and categorical similarity were task-irrelevant features. Also, the number of single target trials was equal to the number of dual target trials.

\section{Experiment 3}

\section{Method}

\section{Participants}

44 volunteers participated in the experiment. Data of one participant were excluded due to very low accuracy (less than 10\%). The final sample consisted of 43 subjects, 30 female, 7 male, 6 participants preferred not to mention their gender. Age varied from 19 to $45(M=23.12$, $S D=5.01$ ), one participant preferred not to mention his age. The link for the experiment was posted on Russian popular social network page, dedicated to jokes about cognitive science “Cognitive Partymaker" (https://vk.com/cogparty). Volunteers were instructed that participants of the experiment can participate in a lottery with the opportunity to win 1000 rubles (around $\$ 14)$ or the set of stickers.

\section{Stimuli}

In this experiment, the stimuli were the following letters of Russian alphabet: $A, 5, B, \Gamma$, Д, 3, Г, П, Р, У. Those letters have different uppercase and lowercase spelling, especially with the font used in the experiment (the same as used in the previous two experiments). Targets and distracters were defined by the orientation - letters with vertical orientation ( 0 grades) were the targets, letters with orientation of 90 grades and 270 grades were the distracters. The color of both targets and distracters could be black or white. In dual target trials, categorical similarity was manipulated as letter identity, perceptual similarity was manipulated as color. The targets were never absolutely identical. Categorically similar targets had the same identity (but not the same spelling). Perceptually similar targets had the same color.

Overall, six conditions were used: categorically similar targets (e.g. A and a, different color), perceptually similar targets (e.g. A and e, same color), categorically and perceptually 
similar targets (e.g. A and a, same color), dissimilar targets (A and e, different color), one target, no targets.

Due to different monitor sizes across the participants, angular size of stimuli are not provided. However, the size of stimuli was 50 pix, the positions varied randomly in $9 * 7$ invisible grid. Two buttons - OK and No - were present at the bottom of the screen. The display was checked on different computers to ensure the stimuli visibility.

\section{Procedure}

Experiment run with Pavlovia software for online experiments (https://pavlovia.org/). Participants used their personal computers (desktop or laptop, but not smartphone or tablet).

Overall, there were 260 trials. Each dual target condition had 30 trials (120 trials overall), single target condition had 120 trials, no target condition had 20 trials. The order of presentation was randomized. Targets and distracters for each trial were chosen randomly from the set of stimuli.

A training session of 10 trials, representing dual target, single target and no target conditions preceded the experiment.

Participants were instructed to search for targets - vertically oriented letters. In dual-target trials, participants subsequently clicked on each target. In single target trials, participants clicked on the target and then on the OK button. In no target trials, participants clicked twice on No button. Overall, the procedure was similar to Experiment 1 and Experiment 2. Example of experimental trial is presented on Fig.11.

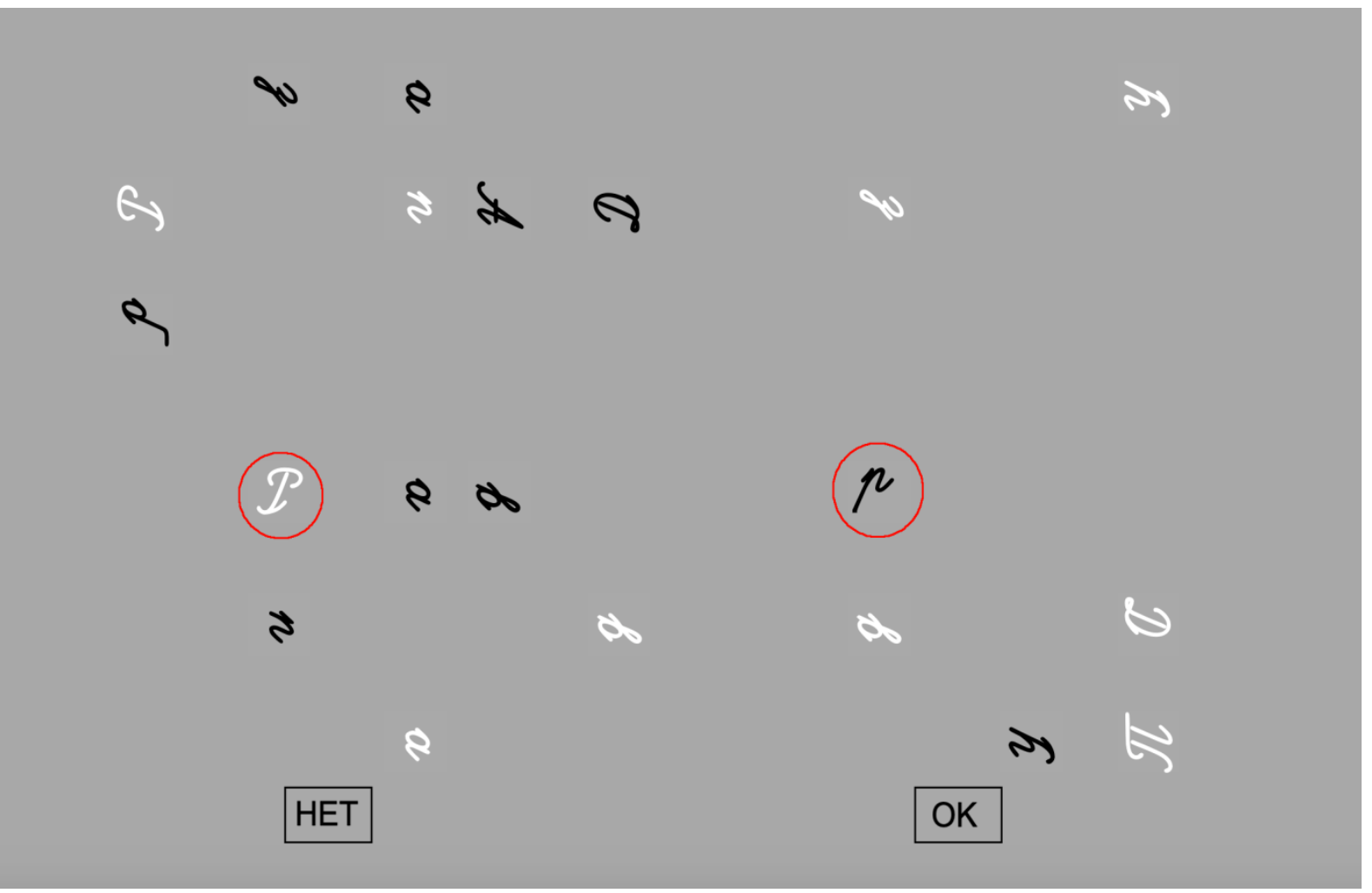

Figure 11. An example of experimental trial in Experiment 3 (condition for two targets, categorical, but not perceptual similarity - targets are marked with red circles) 


\section{Results}

Accuracy and RTs for first and second mouse clicks were analyzed. Accuracy for dualtarget trials was calculated as the percentage of correctly identified second targets to the number of trials, in which at least one target was detected correctly (regardless to the sequence of finding). RTs was analyzed only for correct response trials, RTs more then M+2SD and less then M-2SD were excluded from the analysis.

Data analysis followed the analysis from Experiments 1 and 2. Two types of analysis were made: one way repeated measures ANOVA (factor - type of target, five levels: one target, two dissimilar targets, two perceptually similar targets, two categorically similar targets, two perceptually and categorically similar targets) to reveal the presence of SSM effect if different conditions, and two way repeated measures ANOVA for dual-target conditions, factors perceptual similarity (present or absent) and categorical similarity (present or absent) to reveal the impact of categorical and perceptual similarity for SSM errors. No target condition was not used in the analysis. The Greenhouse-Geisser corrections were applied, when Mauchly's sphericity tests were significant. Pairwise comparisons were made with Bonferroni corrections.

\section{Accuracy}

One-way repeated measures ANOVA revealed the significant effect of type of target factor, $F(2,99)=19.43 ; p<.001 ; \eta p^{2}=.316$. Pairwise comparisons (Bonferroni corrected) revealed the difference between single target condition and two perceptually similar targets condition $(p=.002)$, between single target condition and two dissimilar targets condition $(p<$ .001 ), between single target condition and two perceptually and categorically similar targets condition $(p<.043)$, between two categorically similar targets condition and two perceptually similar targets condition $(p<.001)$, between two categorically similar targets condition and two dissimilar targets condition $(p<.001)$, between two perceptually similar targets condition and two dissimilar targets condition $(p<.001)$, between two perceptually and categorically similar targets condition and two dissimilar targets condition $(p<.001)$.

Two-way repeated measures ANOVA revealed the significant effect of perceptual similarity factor, $F(1,42)=4.56 ; p=.039 ; \eta p^{2}=.098$. The effect of categorical similarity was also significant, $F(1,42)=43.12 ; p<.001 ; \eta p^{2}=.507$. The interaction was significant, $F(1,42)$ $=13.45 ; p=.001 ; \eta p^{2}=.243$. Results are presented in Fig.12-13.

Due to significant interaction, separate rmANOVAs were made for perceptually similar and dissimilar targets (the factor was categorical similarity), as well as separate rmANOVAs were made for categorically similar and dissimilar targets (the factor was perceptual similarity). For perceptually similar targets, the effect of categorical similarity was significant, $F(1,42)=$ $5.51 ; p=.024 ; \eta p^{2}=.116$. For perceptually dissimilar targets, the effect of categorical similarity was also significant, $F(1,42)=39.52 ; p<.001 ; \eta p^{2}=.485$. For categorically similar targets, the 
effect of perceptual similarity was not significant, $F(1,42)=2.90 ; p=.096 ; \eta p^{2}=.065$. For categorically dissimilar targets, the effect of perceptual similarity was significant, $F(1,42)=$ $19.41 ; p<.001 ; \eta p^{2}=.316$.

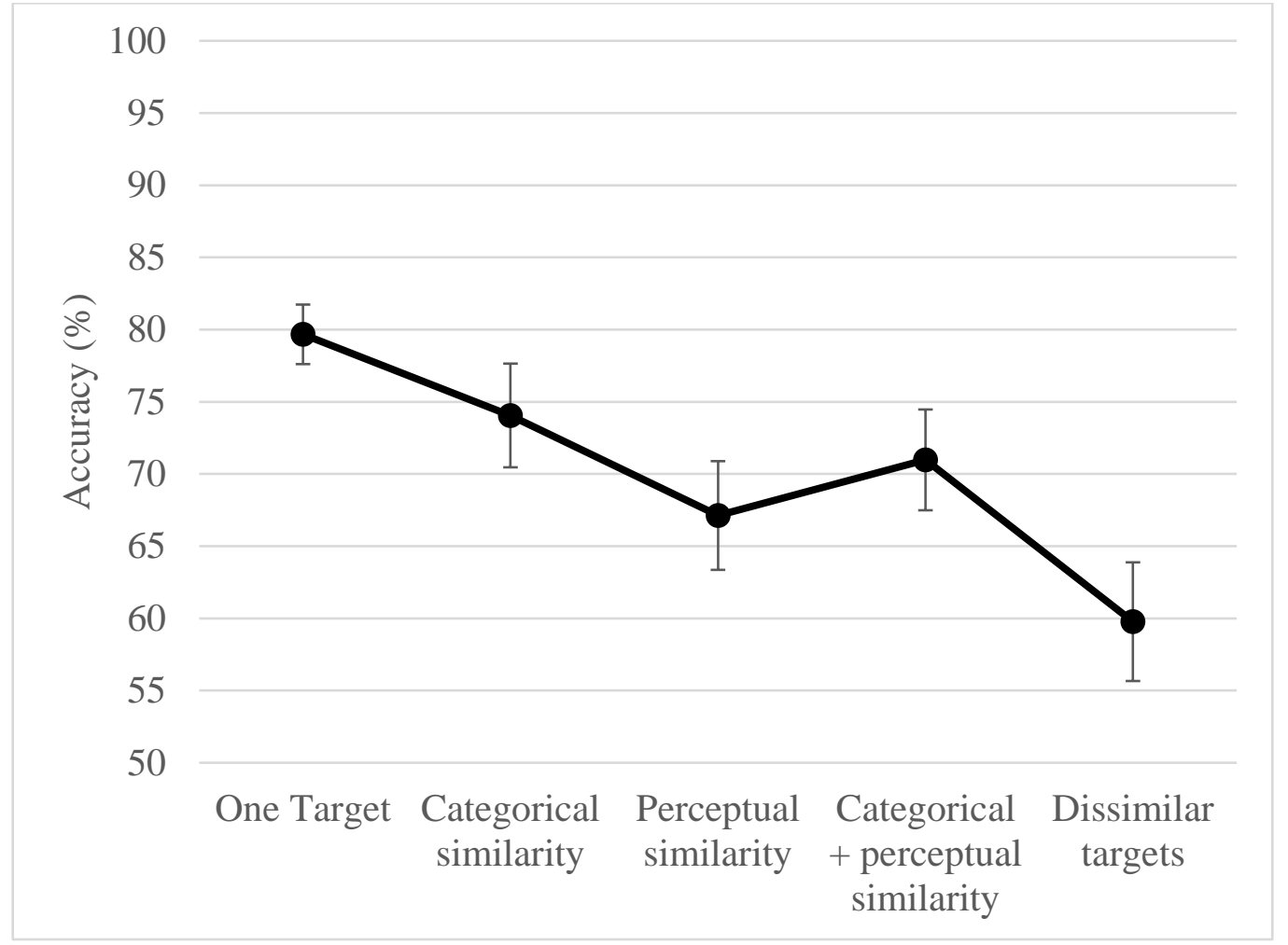

Figure 12. Results of Experiment 3 (accuracy). Error bars represent standard error means

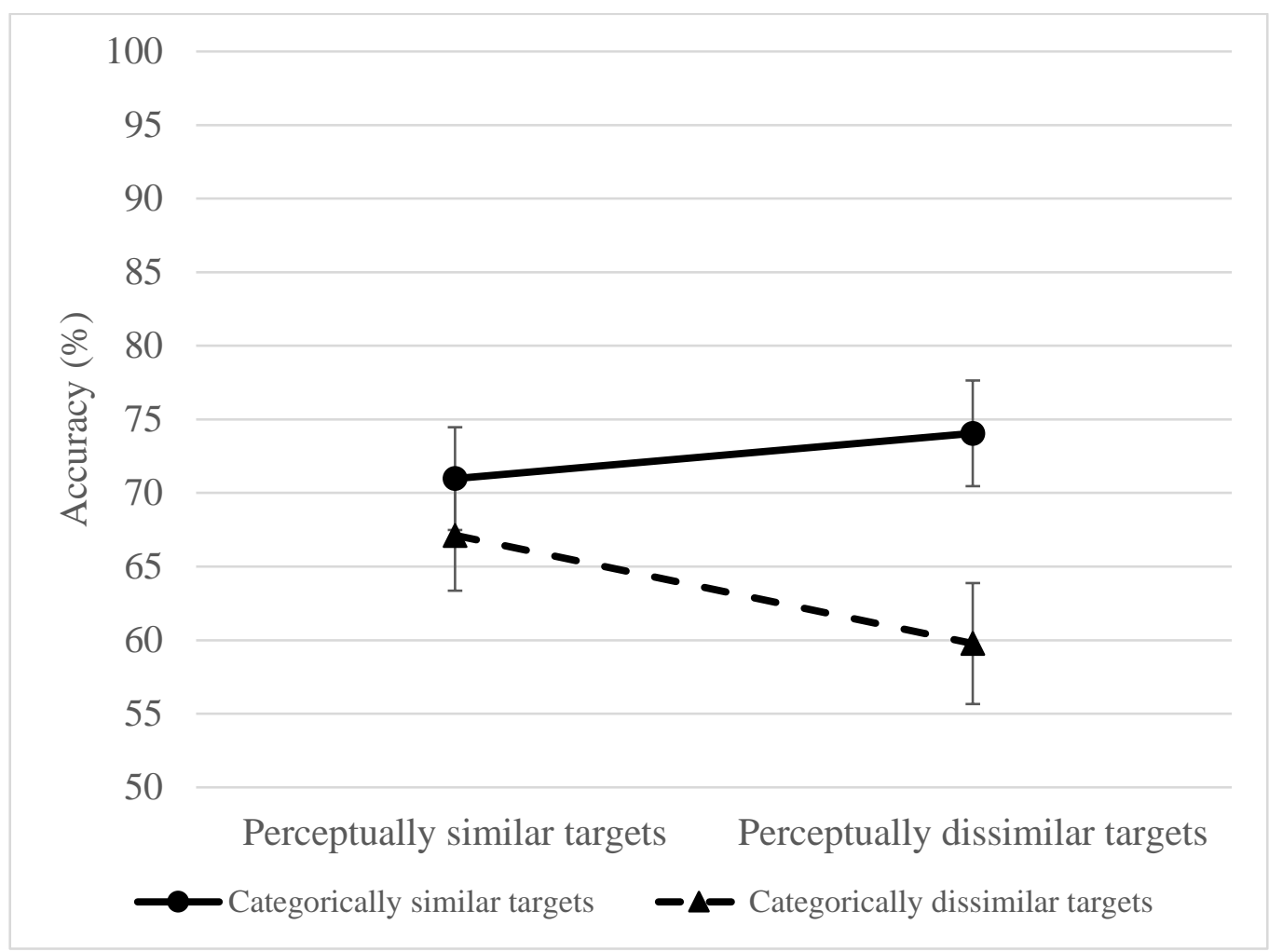

Figure 13. Results of Experiment 3 (accuracy). Error bars represent standard error means 


\section{Reaction time (first click)}

One-way repeated measures ANOVA revealed the significant effect of type of target factor, $F(3,128)=33.87 ; p<.001 ; \eta p^{2}=.452$. Pairwise comparisons (Bonferroni corrected) revealed the difference between single target condition and all dual target conditions $(p<.001)$.

Two-way repeated measures ANOVA revealed the non-significant effect of perceptual similarity factor, $F(1,41)=0.24 ; p=.627 ; \eta p^{2}=.006$. The effect of categorical similarity was also non-significant, $F(1,41)=1.74 ; p=.194 ; \eta p^{2}=.041$. The interaction was non-significant, $F$ $(1,41)=2.91 ; p=.095 ; \eta p^{2}=.066$. Results are presented in Fig. 14 .

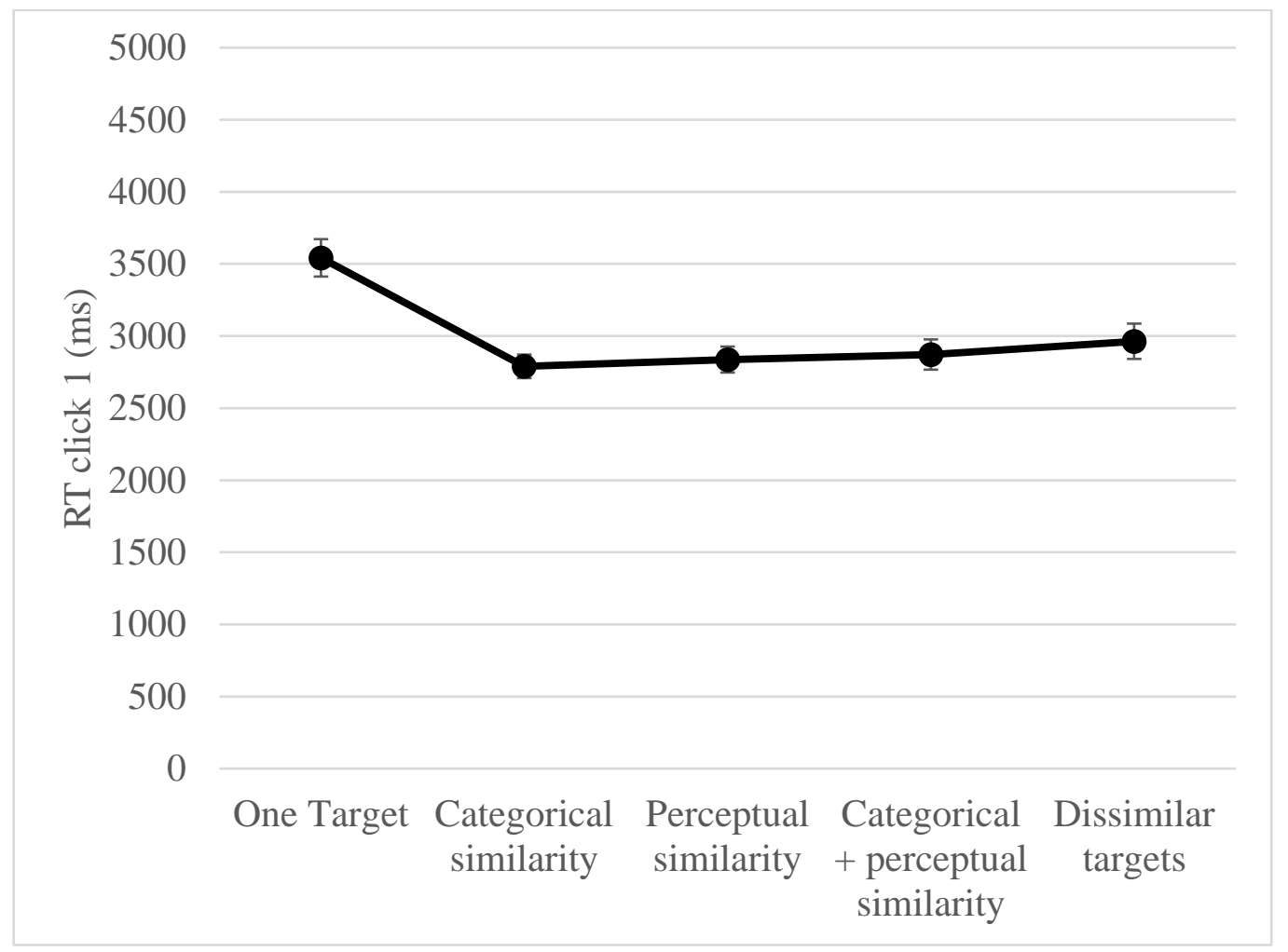

Figure 14. Results of Experiment 3 (RT, first mouse click). Error bars represent standard error means

\section{Reaction time (second click)}

One-way repeated measures ANOVA revealed the significant effect of type of target factor, $F(2,72)=74.4 ; p<.001 ; \eta p^{2}=.650$. Pairwise comparisons (Bonferroni corrected) revealed the difference between single target condition and all dual target conditions $(p<.001)$, between categorically similar targets condition and perceptually similar targets condition $(p=$ $.043)$, between categorically similar targets condition and dissimilar targets condition $(p<.001)$, between perceptually similar targets condition and perceptually and categorically similar targets condition $(p<.001)$, between perceptually and categorically similar targets condition and dissimilar targets condition $(p<.001)$.

Two-way repeated measures ANOVA revealed the significant effect of perceptual similarity factor, $F(1,40)=7.26 ; p=.010 ; \eta p^{2}=.154$. The effect of categorical similarity was 
also significant, $F(1,40)=45.01 ; p<.001 ; \eta p^{2}=.529$. The interaction was not significant, $F$ $(1,40)=0.24 ; p=.629 ; \eta p^{2}=.006$. Results are presented in Fig. 15 .

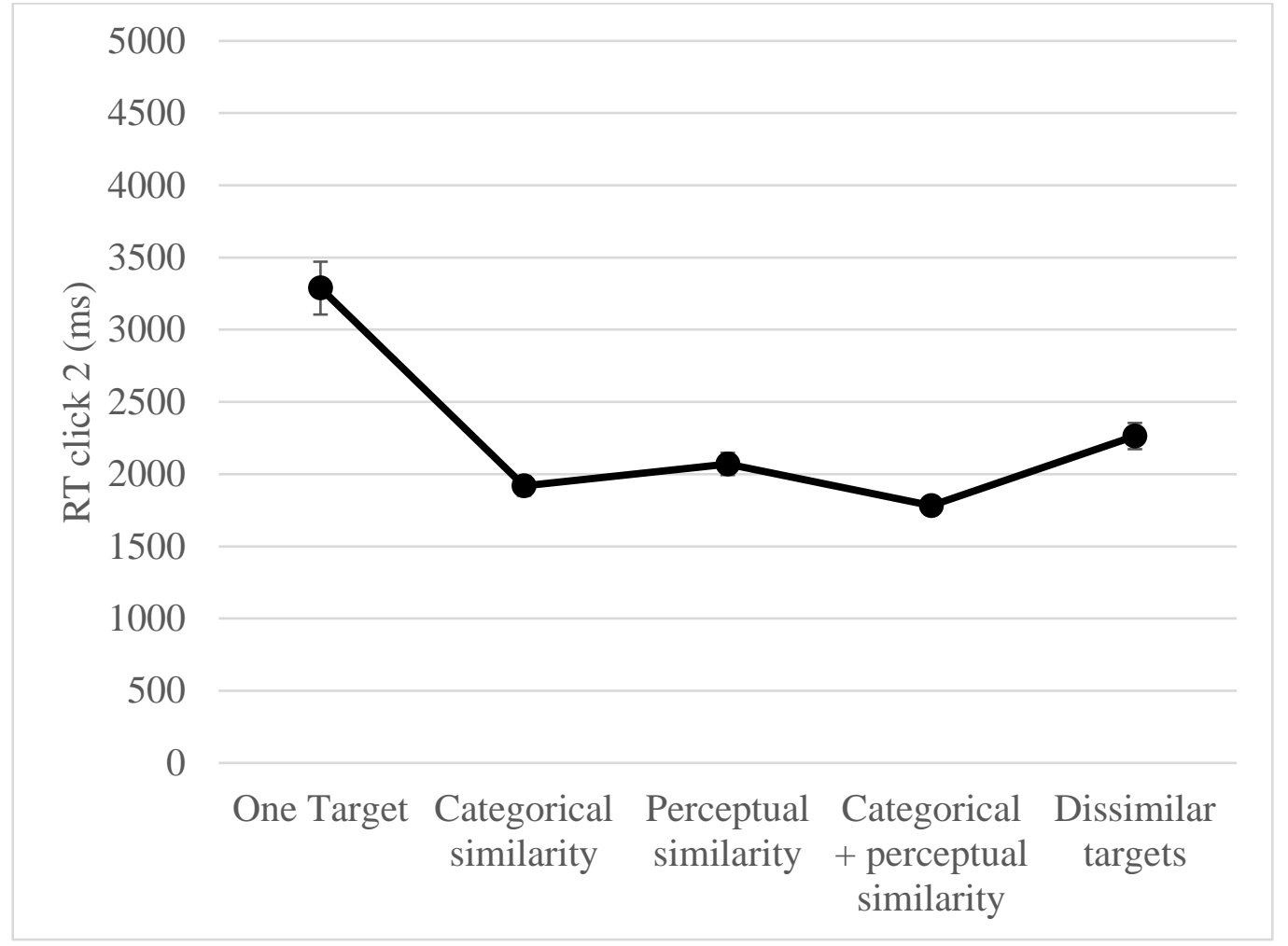

Figure 15. Results of Experiment 3 (RT, second mouse click). Error bars represent standard error means

\section{Discussion}

SSM errors were observed for two dissimilar targets condition, for two perceptually similar targets condition and for two perceptually and categorically similar targets condition. No SSM errors were observed for two categorically similar targets condition. Categorically similar targets were detected better than perceptually similar targets, and perceptually similar targets were detected better then dissimilar targets. Moreover, categorical similarity had the impact on second target detection accuracy regardless of perceptual similarity, whereas perceptual similarity had the impact only for categorically dissimilar targets.

Concerning RTs for the second mouse click, categorically and perceptually similar second targets were found faster than only categorically similar second targets, and only categorically similar second targets were found faster than perceptually similar second targets. The slowest RTs for the second mouse click was observed for dissimilar targets condition. At this point, both perceptual and categorical similarity have the impact on second target detection, but categorical similarity has the upper hand.

Surprisingly, second target detection accuracy for both categorically and perceptually similar targets condition was lower as compared to only categorically similar targets condition, and SSM errors were observed for categorically and perceptually similar targets, but not for only 
categorically similar targets. However, the RTs for the second mouse click assume that both categorically and perceptually similar targets were found faster as compared to only categorically similar targets. One concern may be speed-accuracy trade-off, however, it is not present across other conditions. Another point might be the participants' response bias: in case of high targets similarity, participants could be not completely sure that the second found target is not the same target that they just reported, and thus did not click on it.

RTs for the first mouse click were slower for single target condition and compared to dual target conditions, no difference between dual target conditions was observed. This may be explained by the higher probability to find at least one target when two targets are present on the display as compared to the case when a single target is present. RTs for the second mouse click were also slower for the single target condition as compared to dual target conditions. As the second mouse click in single target condition is the report of second target absence, this result represents the typical visual search pattern for target-absent trials.

Overall, the results of this experiment are consistent with the results of Experiment 1 and Experiment 2, as well as with other studies, where the superiority of categorical similarity over perceptual similarity was observed. Moreover, the priority of categorical similarity over perceptual similarity is maintained when task relevance is controlled.

\section{General discussion}

The main focus of this study was to experimentally separate categorical and perceptual similarity of targets from each other in order to discover their individual contribution to SSM. Letters from Russian alphabet were used as stimuli, and participants searched for vowels among consonants, randomly distributed across computer screen. The main hypothesis was that effect of SSM would be significantly weaker, when targets shared the same category, even though both categorically and perceptually similar targets were expected to make the identification of the second target easier and quicker. These predictions originally came from theories of perceptual and categorical set, which are among the major theoretical foundations for explaining SSM.

It was shown that both perceptual and categorical similarity of targets contribute to the emergence and strength of SSM effect. Still, the most important findings seem to be the confirmation of the superiority of categorical similarity of targets in comparison with perceptual similarity. This was shown in three experiments with thoroughly controlled separation of both these factors. Also, this effect was shown to be independent from task-relevance.

Concerning the theoretical explanations of SSM errors, the most relevant would be the perceptual bias model. This model assumes that participants tend to search for targets that are similar to the first-found target, and to miss the dissimilar ones. Perceptual set mechanisms may 
assume bottom-up priming or top-down working memory guidance. The original idea is related only to perceptual similarity, but results of the current study, taken together with the results of Biggs et al. (2015) consider that conceptual similarity matters as well. Moreover, as categorical similarity has shown to be prevalent over the perceptual similarity, the possible mechanisms of how exactly does the similarity bias work are more likely to be related to working memory guidance than to bottom up priming. At this point, the similarity bias explanation may be integrated with another SSM theory - resource depletion. The hybrid explanation may assume that the representations of the first-found target are uploaded to working memory system, causing both resource depletion and top-down similarity bias.

The possible improvement of this research would be the introduction of real-world objects. However, the limitations of such stimuli, as described above, should be taken into consideration. Nevertheless, the further research in this particular area is of current interest, since the results are very relevant to many practical fields. Visual search for multiple targets is performed on a regular basis by a lot of professionals in various workspaces, for instance, security or medicine. Since it is crucial for them to be precisely accurate and not to make any mistakes, it would be quite helpful to know what exactly causes the visual search mistakes and how to possibly eliminate them, or at least decrease the probabilities of their emergence.

\section{References}

Adamo, S. H., Cain, M. S., \& Mitroff, S. R. (2013). Self-induced attentional blink: A cause of errors in multiple-target search. Psychological Science, 24(12), 2569- 2574.

https://doi.org/10.1177/0956797613497970

Berbaum, K. S., Franken, E. A., Jr., Dorfman, D. D., Rooholamini, S. A.,Coffman, C. E., Cornell, S. H., \& Smith, T. P. (1991). Time course of satisfaction of search. Investigative Radiology, 26, 640-648.

Biggs, A. T., Adamo, S. H., Dowd, E. W., \& Mitroff, S. R. (2015). Examining perceptual and conceptual set biases in multiple-target visual search. Attention, Perception, and Psychophysics, 77(3), 844-855. https://doi.org/10.3758/s13414-014-0822-0

Biggs A.T., Kramer M.R., Mitroff S.R. (2018). Using Cognitive Psychology Research to Inform Professional Visual Search Operations. Journal of Applied Research in Memory and Cognition, 7, 189-198

Cain, M. S., Adamo, S. H., \& Mitroff, S. R. (2013). A taxonomy of errors in multiple-target visual search. Visual Cognition, 21(7), 899-921. https://doi.org/10.1080/13506285.2013.843627 
Cain, M.S., Mitroff, S.R. (2013). Memory for found targets interferes with subsequent performance in multiple-target visual search. The Journal of Experimental Psychology: Human Perception and Performance, 39 (5), 1398 - 1408. doi: 10.1037/a0030726

Collins, A. M., \& Loftus, E. F. (1975). A spreading-activation theory of semantic processing. Psychological Review, 82, 407-428. doi:10.1037/0033-295X.82.6.407

Fleck, M. S., Samei, E., \& Mitroff, S. R. (2010). Generalized —Satisfaction of Search\|: adverse influences on dual-target search accuracy. J Exp Psychol Appl, 16(1), 60-71.

https://doi.org/10.1038/mp.2011.182.doi

Gorbunova, E. S. (2017). Perceptual similarity in visual search for multiple targets. Acta Psychologica, 173, 46-54. https://doi.org/10.1016/j.actpsy.2016.11.010

Kristjansson, A., \& Campana, G. (2010). Where perception meets memory: A review of repetition priming in visual search tasks. Attention, Perception, and Psychophysics, 72(1), 5-18. https://doi.org/10.3758/APP

Kwak, H.-W., Dagenbach, D., Egeth, H. (1991). Further evidence for a time-independent shift of the focus of attention. Perception \& Psychophysics, 49 (5), $473-480$.

Lanina, A. A., \& Gorbunova, E. S. (2018). Stimuli similarity in subsequent search misses.

Experimental Psychology (Russia)Экспериментальная Психология, 11(3), 51-62. https://doi.org/10.17759/exppsy.2018110304

Sall R.J., Feng J. (2019). Dual-target hazard perception: Could identifying one hazard hinder a driver's capacity to find a second? Accident Analysis and Prevention, 131, 213-224

Tuddenham, W. J. (1962). Visual search, image organization, and reader error in roentgen diagnosis. Radiology, 78, 694-704.

Yang, H., \& Zelinsky, G. J. (2009). Visual search is guided to categorically-defined targets. Vision Research, 49(16), 2095-2103. https://doi.org/10.1016/j.visres.2009.05.017

Zhuang, X., Papathomas, T.V. (2011). Cue relevance effects in conjunctive visual search: cueing for location, color, and orientation. Journal of Vision, 11(7). pii: 6

\section{Contact details}

Olga Rubtsova

National Research University Higher School of Economics (Moscow, Russia), Laboratory for Cognitive Psychology of Digital Interfaces Users. Research assistant

olga.rubtsova98@gmail.com

Elena Gorbunova 
National Research University Higher School of Economics (Moscow, Russia), School of Psychology, Laboratory for Cognitive Psychology of Digital Interfaces Users. Associate professor, Laboratory head

gorbunovaes@gmail.com

\section{Financing}

This article is an output of a research project implemented as part of the Basic Research Program at the National Research University Higher School of Economics (HSE University).

\section{Any opinions or claims contained in this Working Paper do not necessarily reflect the views of HSE.}

(C) Rubtsova, Gorbunova, 2020 